Research Article

\title{
Computational Investigation of Flow Control Methods in the Impeller Rear Cavity
}

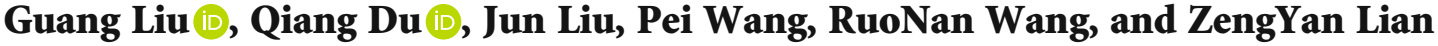 \\ Laboratory of Light-Duty Gas-Turbine, Institute of Engineering Thermophysics, Chinese Academy of Sciences, 11 Beisihuan Road, \\ Beijing 100190, China \\ Correspondence should be addressed to Qiang Du; duqiang@iet.cn
}

Received 27 November 2019; Revised 13 January 2020; Accepted 20 January 2020; Published 22 February 2020

Academic Editor: Angelo Cervone

Copyright (c) 2020 Guang Liu et al. This is an open access article distributed under the Creative Commons Attribution License, which permits unrestricted use, distribution, and reproduction in any medium, provided the original work is properly cited.

\begin{abstract}
In typical median and small aeroengines, the air used to realize the functions such as cooling of turbine blades and disks, sealing of turbine cavities and bearing chambers, adjusting of rotating assembly axial load is normally drawn through the rear cavity of centrifugal impeller, so the thorough understanding of flow characteristics and pressure distribution and the proposal of the corresponding control methods in the cavity are the key to design the rational secondary air system. With an impeller rear cavity in a small turbofan engine as an object, the current study was dedicated to the investigation of flow control methods in the cavity. Two methods, namely, baffle and swirl-controlled orifice, were proposed to regulate the pressure loss and distribution in the cavity. Furthermore, the influence of geometry parameters of the two methods such as the length of baffle, the space between the baffle and rotating disk wall, the orientation, and radial position of swirl-controlled orifice was investigated. The CFD results show that the swirl-controlled orifice which could deswirl the flow is more effective in regulating the pressure loss and its distribution in cavity than baffle. The variation of the radial position of the swirl-controlled orifice had little influence on pressure loss but obvious influence on pressure distribution; therefore, decreasing the radial position could reduce the axial load on the rotating disk without changing the outlet pressure.
\end{abstract}

\section{Introduction}

During the aeroengine design process, the impeller rear cavity differs broadly in the parameters of inlet/outlet conditions because of the different centrifugal compressor and turbine aerodynamic designs and the different demands to ensure adequate cooling of turbine blades and disks, sealing of turbine cavities and bearing chambers, and adjusting of rotating assembly axial load. Furthermore, in the aeroengine test process, the deviation between the true matching point of the whole machine and the design matching point will lead to the deviation of pressure distribution in the impeller rear cavity and thus causes the failure of the functions. Hence, the accurate prediction of the pressure distribution and the proposal of the corresponding control methods in the impeller rear cavity have great significance in the optimization of the secondary air system of aeroengines.
A considerable amount of work about the flow characteristics in the rotor-stator cavity with a centripetal inflow has been carried out by a number of workers [1-6]. The results show that for a rotor-stator cavity with centripetal flow, the internal flow conforms to the Batchelor type which consisted of an inviscid core and two Ekman type boundary layers. In the core region, the axial velocity and radial velocity are negligibly small, so the equation of the tangential velocity in the NS equation is simplified to the balance of the centrifugal force and the radial pressure gradient. Thus, the radial distribution law of the pressure in the cavity is determined by the tangential velocity distribution in the core region. EI-Oun et al. [7] used the pitot tube measurement to obtain the velocity distribution in the rotor-stator cavity with a centripetal flow. Pincombe [8] used the LDV flow field visualization method to measure the velocity profiles in the cavity under different inlet swirl ratio $\beta_{0}$ and different rotational Reynolds number $\operatorname{Re}_{\varphi}$. It is found that the value of $\beta_{0}$ has a 

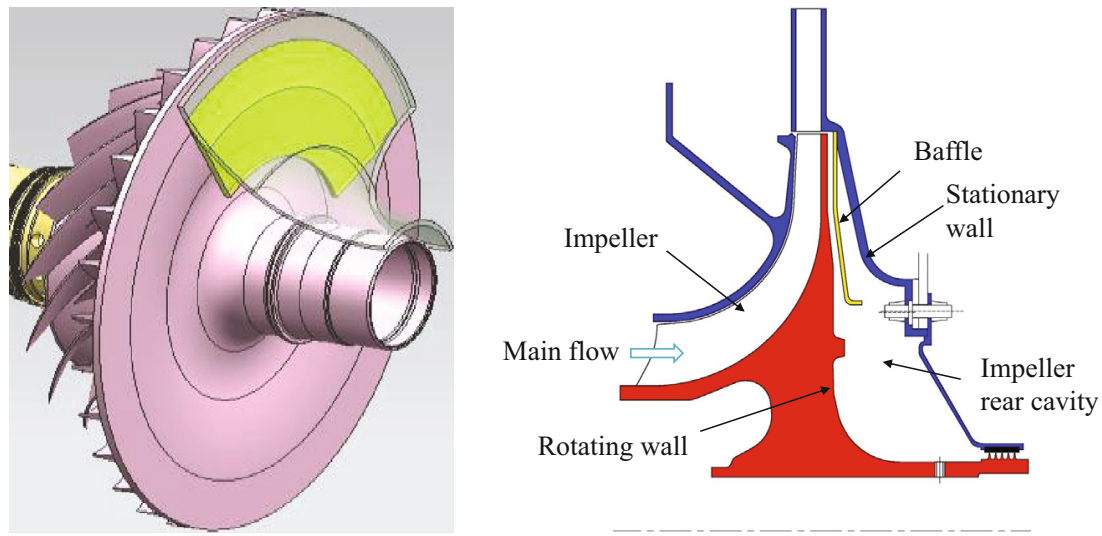

(a) The impeller rear cavity with baffle
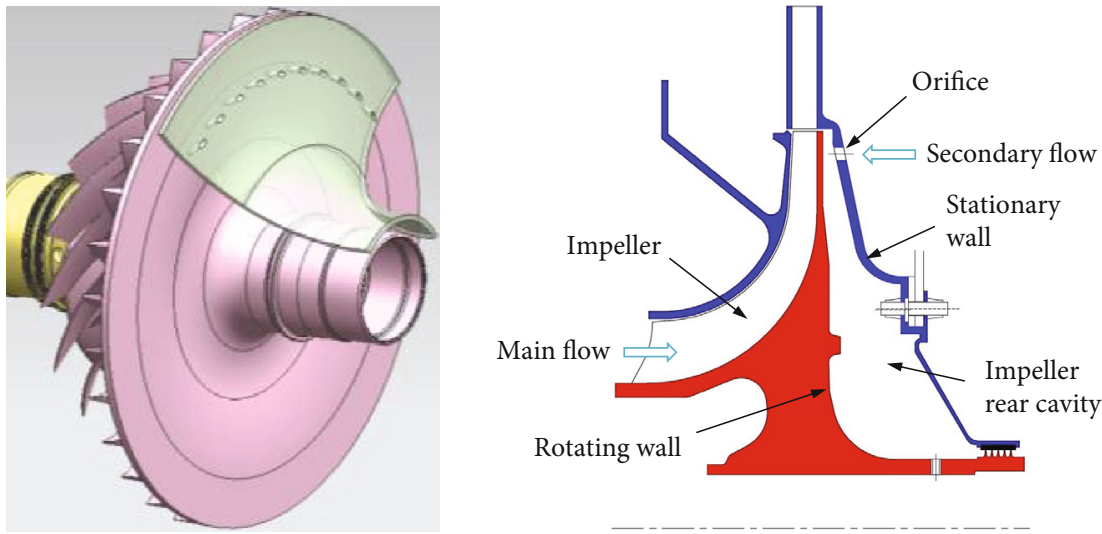

(b) The impeller rear cavity with swirl-controlled orifice

FIGURE 1: Impeller rear cavity with control method.

significant influence on the distribution of the swirl ratio $\beta$ in the central core region. Hart and Turner $[9,10]$ used numerical and experimental methods to obtain the pressure and swirl ratio in the impeller rear cavities with different geometries and air bleed flow. The aim of their researches was to obtain a series of curves that can help predict velocity and pressure distribution quickly and accurately without the need for costly CFD work or rig testing during the initial engine design phase. Through the above researches, it is found that the regulation of the pressure distribution in the impeller rear cavity is to regulate the tangential velocity distribution.

Three types of flow control methods used to regulate the tangential velocity distribution in the rotating cavity are investigated in previous papers: fin [11], deswirl nozzle $[12,13]$, and tube [14] vortex reducers. For fin vortex reducer, the flow with high tangential velocity gets through the diversion passage between rectangular fins that are axial-symmetrically mounted and extended radially on the disks. For deswirl nozzle vortex reducer, the flow gets through the angled nozzles that are installed on the rotating disks. Because the nozzles are angled, the direction of the air from the outlet of nozzles could be varied from 0 to $180^{\circ}$ theoretically by altering the angle. For tube vortex reducer, the flow gets through the axial-symmetrically installed radial tubes from the compressor blade platforms to the disk bores with constant swirl ratio.

However, because of the structure limitation, all the above three vortex reducers are not suitable for the rotorstator cavity. Therefore, in the present paper, two methods, namely, baffle and swirl-controlled orifice, were proposed to regulate the pressure loss and distribution in the rotorstator cavity: (1) a baffle, shown in Figure 1(a), is installed between the rotating and stationary walls. More detailed introduction about baffle can be seen in the author's previous paper [15]. (2) Swirl-controlled orifice is shown in Figure 1(b). The stationary wall in the cavity can be provided with a plurality of orifices through which the impeller rear cavity is in fluid communication with the combustion section such that the pressurized air around the combustor is directed into the impeller rear cavity. The orifices extend axially and tangentially in a direction substantially the same or opposite to the tangential velocity of the pressurized air flow to direct the air flow from the combustion section there through into the impeller rear cavity in a direction substantially the same or opposite to the tangential direction of the pressurized air flow entering the impeller rear cavity at the impeller tip. Therefore, the angular momentum of both pressurized air flows will act on each other to change the angular momentum of the total pressurized air contained within the 


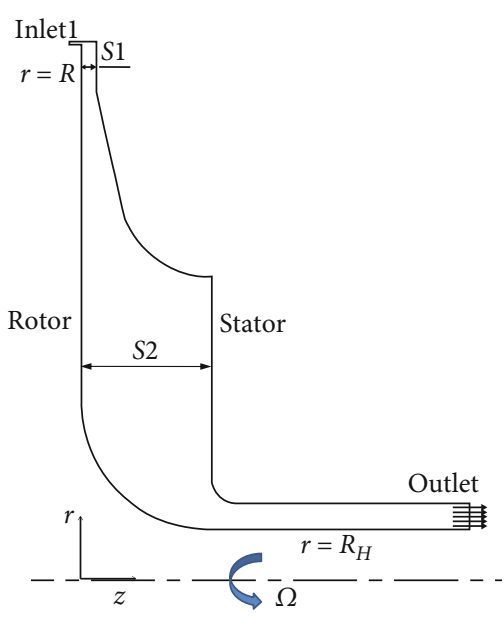

(a)

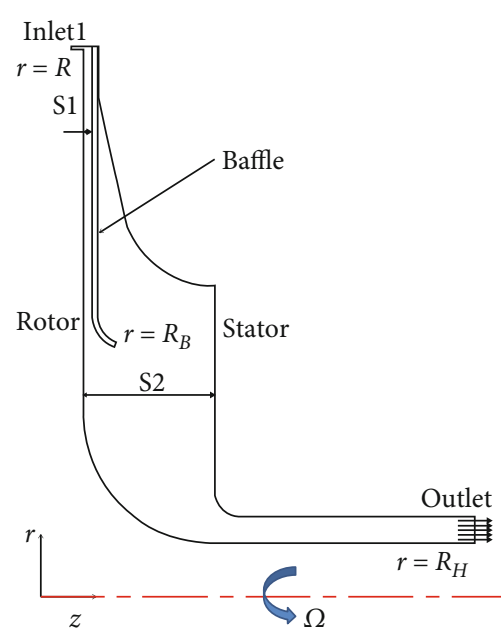

(b)

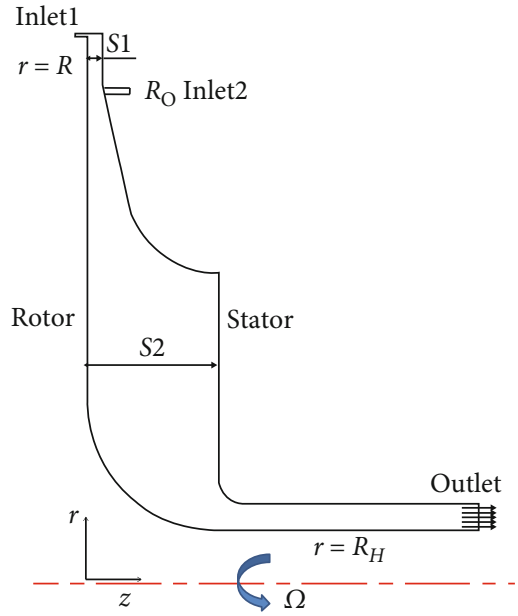

(c)

FIGURE 2: (a) The cavity without control method. (b) The cavity with baffle. (c) The cavity with swirl-controlled orifice.

impeller rear cavity and thus the radial static pressure gradient. The numerical simulations were conducted for various $\beta_{0}(0 \sim 0.8), \lambda_{\mathrm{T}}(0.028 \sim 0.280)$ with and without control methods to investigate the control mechanisms of these two methods.

\section{The Numerical Models}

2.1. Geometry. The structures of the original cavity, the cavity with baffle, and the cavity with swirl-controlled orifice are, respectively, shown in Figures $2(\mathrm{a})-2(\mathrm{c}) . R_{\mathrm{H}}$ is the inner radius of rotating disk, and $\boldsymbol{R}$ is the outer radius; $R_{\mathrm{B}}$ is the inner radius of baffle; the axial distance between rotating and stationary disks varies from $S \mathbf{1}$ to $S 2$; the radius of axis of the orifice is $R_{\mathrm{O}}$; the diameter of the orifice is $D$; the number of the orifice is $\boldsymbol{X}$; the angle between the axial direction of the orifice and the tangential direction is $\boldsymbol{\alpha}$ (when the holes extend in a direction substantially opposite to the rotating direction of the rotor, $\boldsymbol{\alpha}$ is positive). Table 1 shows the values of all the geometric parameters.

2.2. Numerical Method. Steady state simulations were performed using commercial code CFX v13 with the k- $\omega$-SST turbulence model and wall function approach. A periodic condition was imposed so that an axisymmetric 3D sector was modelled.

Different grid sizes were used to study the grid independency for the cavity with and without control methods. The calculation results show that the variation of outlet pressure coefficient is less than $2 \%$ for cases with different grid sizes. At last, three meshes with 480000,900000 , and 2000000 cells are chosen for the model in Figures 2(a)-2(c) separately. The first cell size of the wall is $1 \times 10^{-6} \mathrm{~m}$ for all the three models; for the cavity with swirl-controlled orifice, the O-type mesh is used in the orifice. The wall $y^{+}$values of all the numerical cases are smaller than 3 and suitable for the wall functions. Figure 3 shows the mesh model for the cavity with swirlcontrolled orifice.
TABLe 1: Geometric parameters.

\begin{tabular}{lc}
\hline$R(\mathrm{~mm})$ & 144 \\
$R_{\mathrm{H}}(\mathrm{mm})$ & 48 \\
$R_{\mathrm{O}}(\mathrm{mm})$ & 132 \\
$D(\mathrm{~mm})$ & 0.9 \\
$X$ & 30 \\
$\alpha$ & 2.9 (without baffle) \\
$S 1(\mathrm{~mm})$ & 1.9 (with baffle) \\
$S 2(\mathrm{~mm})$ & 25.7 \\
$G 1=S 1 / R$ & 0.02 (without baffle) \\
$G 2=S 2 / R$ & 0.0132 (with baffle) \\
\hline
\end{tabular}

2.3. Boundary Conditions and Dimensionless Parameters. All the walls of the cavity are set to adiabatic and no slip condition. For cases in the cavity with swirl-controlled orifice, the mass flow rate ratio of inlet 2 and inlet 1 is set to $0.1 / 0.2 / 0.3$. The values of dimensionless parameters and boundary conditions for the numerical cases in the current paper are listed in Table 2. In recent years, many researchers such as Liu et al. [15] find that for any impeller rear cavity, when $\beta_{0}$ and $\lambda_{\mathrm{T}}$ are the same, the difference of $C_{\mathrm{p}}$, between the conditions with different combination of $C_{\mathrm{w}}$ and $\operatorname{Re}_{\varphi}$, is ignorable. Therefore, the dimensionless flow parameters discussed in the current paper are $\beta_{0}$ and $\lambda_{\mathrm{T}}$.

2.4. Verification of the Numerical Model. The experimental data from Poncet et al. [5] in a rotor-stator cavity with centripetal throughflow was chosen for verification. The rotorstator cavity consisted of a smooth stationary disk of $506 \mathrm{~mm}$ diameter and a smooth rotating disk of $500 \mathrm{~mm}$ diameter, shown in Figure 4. The distance between the stationary and rotating disks can be changed from 3 to $12 \mathrm{~mm}$. The rotating disk is driven by a $5.5 \mathrm{~kW}$ electric servo motor. 


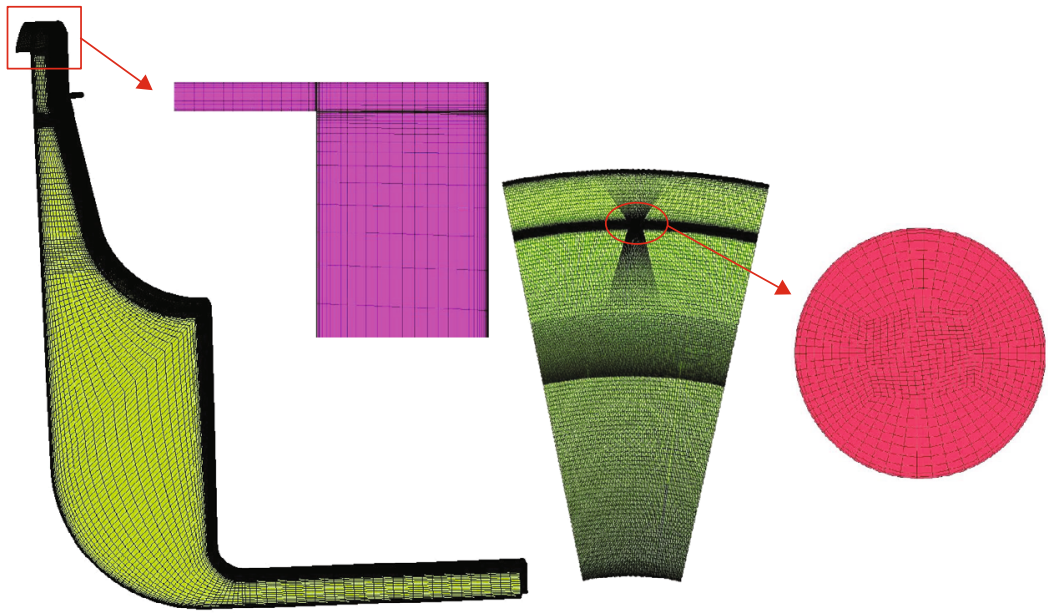

FIGURE 3: The CFD mesh for the cavity with swirl-controlled orifice.

TABLE 2: The values of parameters.

\begin{tabular}{lc}
\hline Location & $\begin{array}{c}\text { Conditions } \\
\text { Inlet1 }\end{array}$ \\
Tnlet2 & $\begin{array}{c}\text { Total pressure: } P_{\mathrm{t}}=600000 \mathrm{~Pa} \\
\text { Temperature: } T_{\mathrm{t}}=479 \mathrm{~K}\end{array}$ \\
Outlet & $\begin{array}{c}m_{2} /\left(\mathrm{m}-\mathrm{m}_{2}\right)=\mathrm{Ra}=0.1 / 0.2 / 0.3 \\
\text { Mass flow rate, } m: 25.8 \sim 253(\mathrm{~g} / \mathrm{s})\end{array}$ \\
Walls & Adiabatic and no slip \\
\hline Parameters & Range \\
Rotational speed of & $5000 \sim 30000(\mathrm{rpm})$ \\
rotating wall, $N$ & $0 \sim 0.8$ \\
$\beta_{0}$ & $1.83 \times 10^{6} \sim 1.11 \times 10^{7}$ \\
Re ${ }_{\varphi}$ & $6.9 \times 10^{3} \sim 4.8 \times 10^{4}$ \\
$C_{\mathrm{w}}$ & $0.028 \sim 0.280$ \\
$\lambda_{\mathrm{T}}$ &
\end{tabular}

Because the flow in the cavity is water, the cavity pressure is maintained at 2 bar by a buffer and two pressure sensors to avoid cavitation. The temperature of the water is kept constant $\left(23^{\circ} \mathrm{C}\right)$ by a heat exchanger to achieve the unchanged density $\boldsymbol{\rho}$ and dynamic viscosity $\boldsymbol{\mu}$. The water enters the cavity through 48 angled holes of $10 \mathrm{~mm}$ diameter and thus has preswirl. The mean inlet swirl ratio $\beta_{0}$ of the flow in prerotation ranges from 0.43 to 0.54 .

The tangential and radial velocities in the cavity are measured by a two-dimension laser Doppler anemometer. The pressure on the stator at the dimensionless radial positions $0.186,0.22,0.28,0.34,0.4$, and 0.46 is measured by six piezoresistive transducers. The comparison here is for two different throughflow bleeds $\left(C_{\mathrm{w}}=5929,9881\right)$ with an axial gap of $9 \mathrm{~mm}$ and $\operatorname{Re}_{\varphi}=1.0 \times 10^{6}$. The inlet swirl ratio $\beta_{0}$ is 0.5 .

A $4^{\circ}$ sector mesh shown in Figure 5 and the same numerical software as above were chosen for the verification. The structured mesh was chosen, and the first cell size of the wall

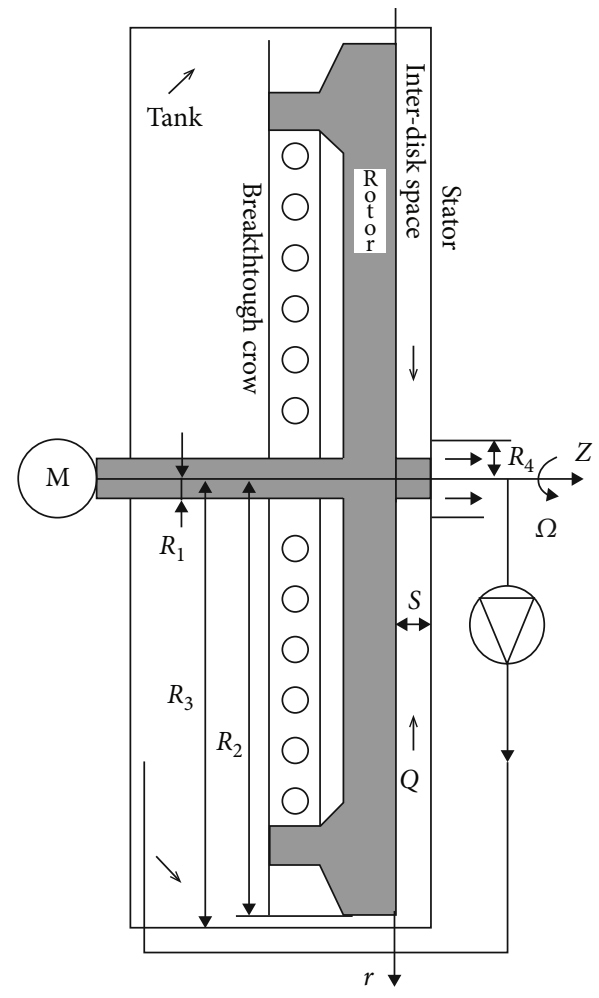

Figure 4: Schematic diagram of verification case.

is $2 \times 10^{-6} \mathrm{~m}$. The mesh with 327229 cells was used for the model after the grid independence research. The verification test was carried out using the different turbulent models including Spalart-Allmaras, standard k- $\varepsilon$, and SST k- $\omega$. The results using the SST k- $\omega$ model were found to show the best agreement.

At the inlet, the total temperature, total pressure, and flow velocity direction were set. At the outlet, the mass flow rate was set. At all the walls, the adiabatic and no slip condition was set. Figures 6(a) and 6(b) compare the current computational results for the radial variation of static pressure 


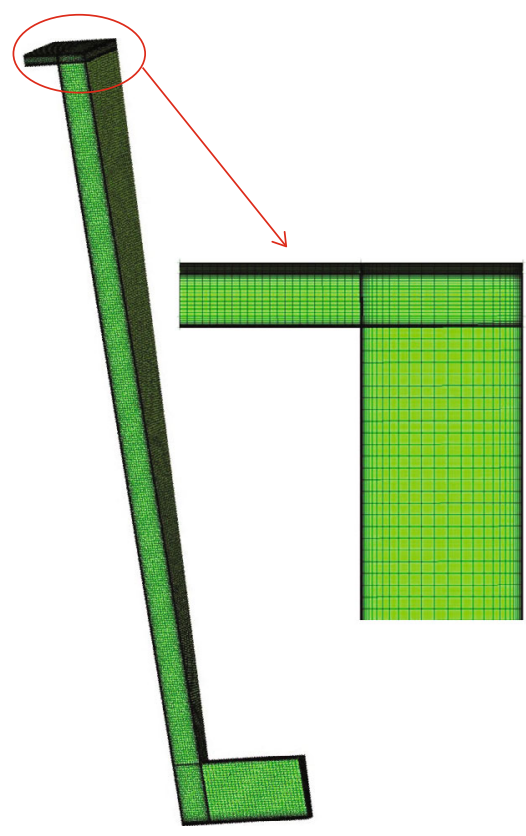

FIgURE 5: The mesh for verification case.

coefficient $C_{\mathrm{p}}$ and swirl ratio $\beta$ with the experimental data of Poncet. The CFD results agree well with the experimental data.

\section{Numerical Results and Discussions}

\subsection{The Effect of the Baffle}

3.1.1. The Effect on the Flow Structure and Pressure Loss. The effect of the baffle on the flow structure and pressure loss has been carried out in the author's previous research [15]. The results show that the biggest difference of flow characteristic in the cavity with and without baffle is that a big counterclockwise vortex exists in the stationary cavity composed of the stationary wall and the baffle surface. The existence of the big counterclockwise vortex leads to the diminution of the average swirl ratio of cavity. Thus, the pressure loss of cavity with the baffle reduces. More detailed analysis can be found in research [15].

3.1.2. The Influence of Geometry Parameters of the Baffle. It is found by investigation in previous research that the baffle can reduce the outlet pressure loss of the impeller rear cavity, but whether it is any length, any space between the baffle and rotating disk wall, can the baffle play a role in reducing pressure losses? How to better design the baffle needs further research on the influence of the length of the baffle and the axial space between the baffle and rotating disk wall.

(1) The Length of the Baffle. Figures 7(a) and 7(b) show the variation of $\beta_{\text {outlet }}$ and $C_{\mathrm{p} 1}$ with $\lambda_{\mathrm{T}}$ when $\beta_{0}=0.8$ of the cavity without and with three different baffles whose nondimensional lengths $L^{*}\left(L^{*}=1-R_{\mathrm{B}} / R\right)$ are $0.264,0.403$, and 0.542 , respectively.
As shown in Figure 7(a), when the length of the baffle is very short $\left(L^{*}=0.264\right), \beta_{\text {outlet }}$ is the same as when there is no baffle; as the length of the baffle increases, $\beta_{\text {outlet }}$ gradually decreases. As shown in Figure $7(\mathrm{~b})$, when $L^{*}=0.264, C_{\mathrm{p} 1}$ is also the same as when there is no baffle; when $L^{*}$ increases from 0.264 to $0.403, C_{\mathrm{p} 1}$ reduces; however, when $L^{*}$ increases from 0.403 to $0.542, C_{\mathrm{p} 1}$ increases instead, which is different from the change rule of $\beta_{\text {outlet}}$.

The swirl ratio contours and streamlines in $r$ - $z$ section of the cavity with three different baffles of different lengths under the condition $\lambda_{\mathrm{T}}=0.28, \beta_{0}=0.8$ are shown in Figure 8. It can be seen from Figure 8 that when the length of the baffle is very short $\left(L^{*}=0.264\right)$, the stationary cavity formed by the stationary wall and the baffle is too small to cause great impact on the mainstream; the flow structure is essentially the same as the cavity without baffle; therefore, when $L^{*}=0.264, \beta_{\text {outlet }}$ and $C_{\mathrm{p} 1}$ of the impeller rear cavity are the same as the cavity without baffle; when $L^{*}$ increases from 0.264 to 0.403 , a large static cavity is formed between the baffle and the stationary wall; the main air flowing out from the small clearance mixes vigorously with the counterflow in the static cavity and leads to the diminution of $\beta_{\text {outlet; }}$ when $L^{*}$ continues to increase from 0.403 to 0.542 , the static cavity becomes larger; the reduction of $\beta_{\text {outlet }}$ caused by the mixing is more pronounced because of the increase of the recirculating flow rate in the static cavity.

The above analysis can be verified from the comparison of the radial distribution of the average $\beta$ in cavity with different baffles of different $L^{*}$ under the condition $\lambda_{\mathrm{T}}=0.28$ and $\beta_{0}=0.8$, as shown in Figure 9(a). When $L^{*}=0.264$, the variation of $\beta$ does not show the sudden decrease; when $L^{*}=0.403,0.542, \beta$ is significantly reduced at the exit position of the baffle, and the decrease is more noticeable when $L^{*}=0.542$.

Figures 9(b) and 9(c) show the comparison of the radial variation of $C_{\mathrm{p}}$ and nondimensional radial velocity $V_{\mathrm{r}}{ }^{*}$ in cavity with different baffles of different $L^{*}$ under the condition $\lambda_{\mathrm{T}}=0.28$ and $\beta_{0}=0.8$. When $L^{*}=0.264$, there is no sudden decrease of $C_{p}$, which is consistent with the change rule of $\beta$; when $L^{*}$ increases from 0.264 to 0.403 , the mixing happens at the outlet of the baffle; therefore, $\beta$ decreases and results in a decrease of $C_{\mathrm{p}}$; when $L^{*}$ continues to increase from 0.403 to $0.542, \beta_{\text {outlet }}$ is further lowered, but $C_{\mathrm{p} 1}$ increases instead. The reason why the variations of $\beta_{\text {outlet }}$ and $C_{\mathrm{p} 1}$ are inconsistent is that when $L^{*}$ increases from 0.403 to 0.542 , in the radial region from $L^{*}=0.403$ to $L^{*}=0.542, G=S / R$ of the cavity with baffle $L^{*}=0.542$ is 0.02 and much smaller than that of the cavity with baffle $L^{*}=0.403(G=0.18)$; hence, for the large flow rate conditions (corresponding to large $\lambda_{\mathrm{T}}$ ), the radial velocity is greatly increased and cannot be ignored (shown in Figure 9(c)). At this time, the variation of static pressure is codetermined by the change of $\beta$, the radial velocity, and the hydraulic friction caused by the nonnegligible radial velocity. Since the radial velocity is gradually increased during the inward flow process and the large 


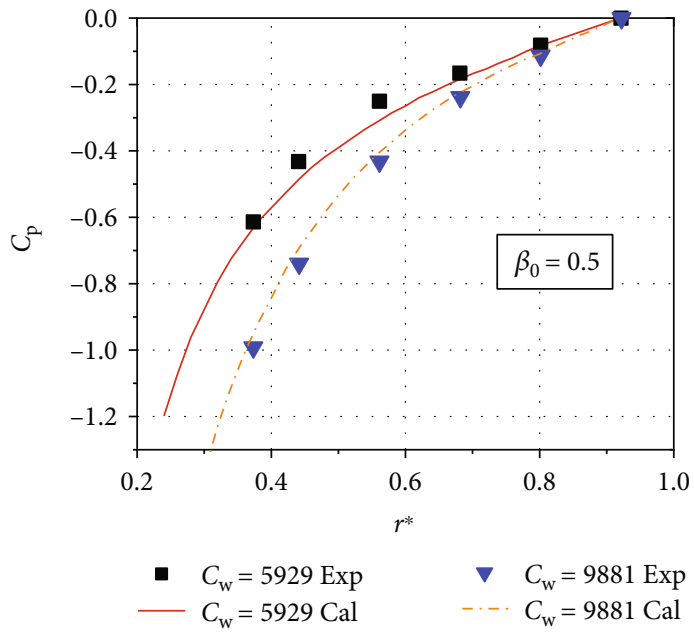

(a) $C_{\mathrm{p}}$

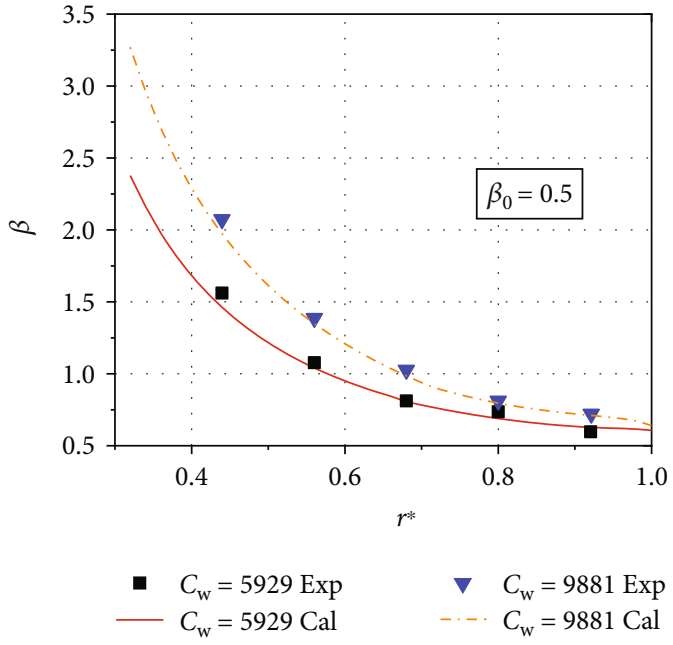

(b) $\beta$

FIgURE 6: Comparison of radial variation of $C_{\mathrm{p}}$ and $\beta$ between numerical results using SST turbulence model and experimental data.

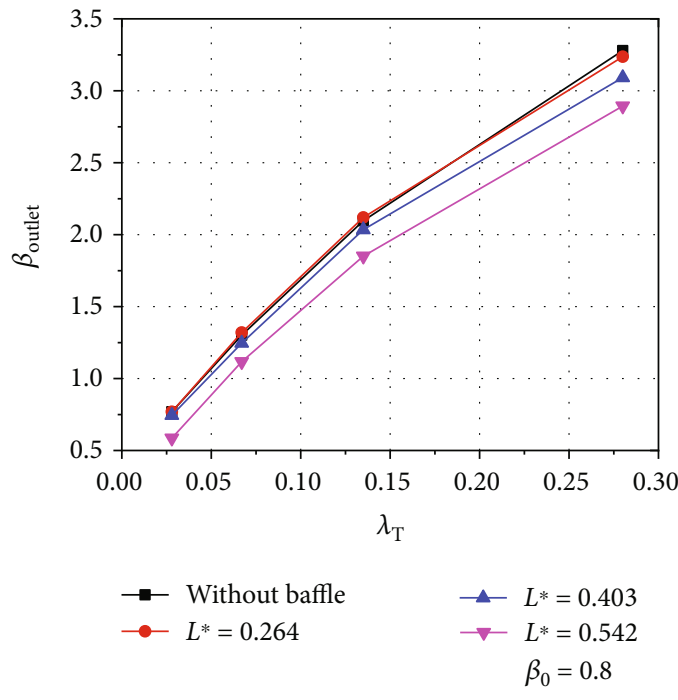

(a) $\beta_{\text {outlet }}$

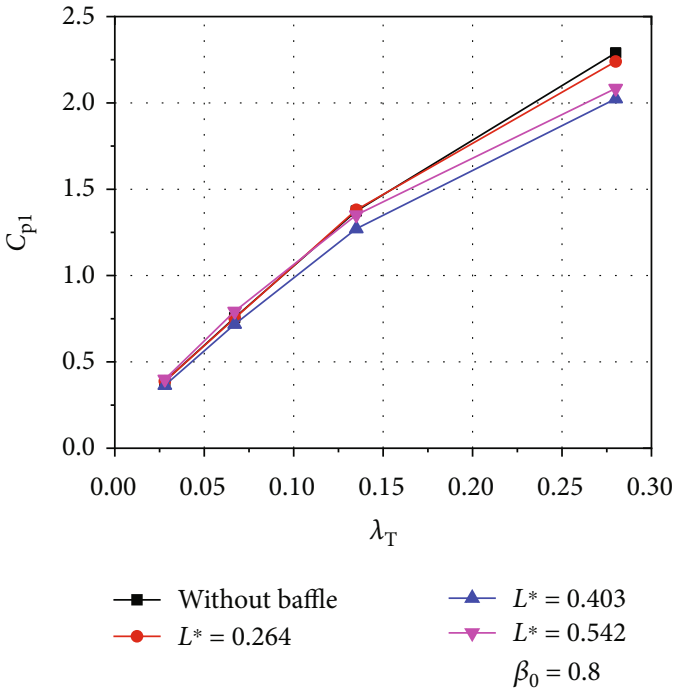

(b) $C_{\mathrm{p} 1}$

Figure 7: The variation of $\beta_{\text {outlet }}$ and $C_{\mathrm{p} 1}$ with $\lambda_{\mathrm{T}}$ when $\beta_{0}=0.8$ of the cavity without and with three different baffles of different lengths.

radial velocity causes the hydraulic friction loss, when $L^{*}$ increases from 0.403 to 0.542 , although $\beta_{\text {outlet }}$ reduces, $C_{\mathrm{p} 1}$ increases due to the increase of radial velocity.

In summary, as the nondimensional length of the baffle increases, $\beta_{\text {outlet }}$ is gradually reduced, but $C_{\mathrm{p} 1}$ is first reduced and then increased. Therefore, when designing the baffle, there is an optimal length to make the static pressure loss in the cavity the smallest.

(2) The Space between the Baffle and Rotating Disk Wall When $L^{*}=0.403$. Figures 10 (a) and $10(\mathrm{~b})$ show the variation of $\beta_{\text {outlet }}$ and $C_{\mathrm{p} 1}$ with $\lambda_{\mathrm{T}}$ when $\beta_{0}=0.8$ of the cavity without and with four different baffles whose nondimensional space between the baffle and rotating disk wall $G 1(G 1=S 1 / R)$ is $0.02,0.013,0.006$, and 0.003 , respectively.
As shown in Figure $10(\mathrm{a})$, when $G 1=0.02, \beta_{\text {outlet }}$ is smaller than when there is no baffle; when $G 1$ reduces from 0.02 to $0.006, \beta_{\text {outlet }}$ is basically unchanged; when $G 1$ continues to decrease to $0.003, \beta_{\text {outlet }}$ is slightly reduced. In Figure $10(\mathrm{~b})$, when $G 1=0.02, C_{\mathrm{p} 1}$ is smaller than when there is no baffle; when $G 1$ reduces from 0.02 to $0.013, C_{\mathrm{p} 1}$ is unchanged; when $G 1$ reduces from 0.013 to $0.006, C_{\mathrm{p} 1}$ increases; when $G 1$ continues to decrease to $0.003, C_{\mathrm{p} 1}$ increases significantly and is much larger than that of the cavity without and with other baffles. In addition, the larger $\lambda_{\mathrm{T}}$ is, the more intensely $C_{\mathrm{p} 1}$ changes with $G 1$. Through comparison between Figures $10(\mathrm{a})$ and $10(\mathrm{~b})$, it is found that the increase in outlet static pressure loss that occurs when $G 1$ reduces to 0.003 is not caused by the increase of the swirl ratio. 


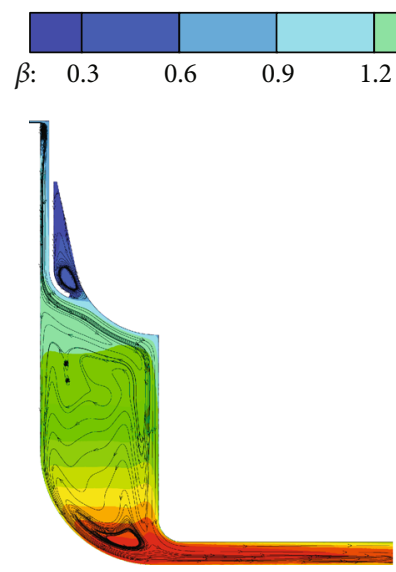

(a) $\mathrm{L}^{*}=0.264$

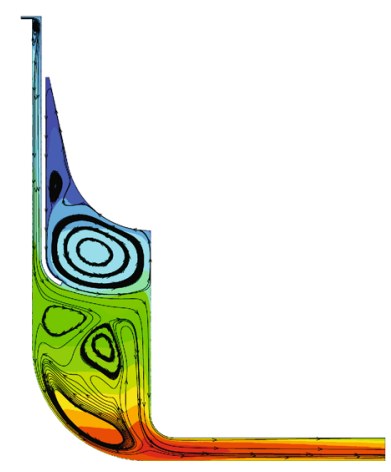

(b) $\mathrm{L}^{*}=0.403$

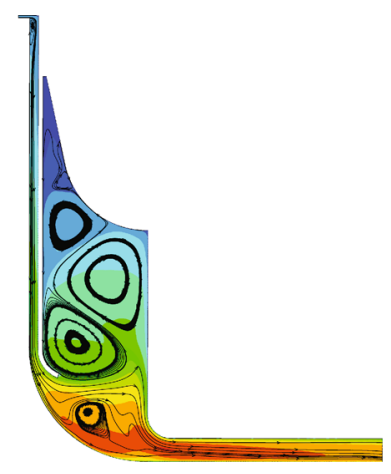

(c) $\mathrm{L}^{*}=0.542$

FIGURE 8: The swirl ratio contours and streamlines in $r-z$ section of the cavity with three different baffles of different lengths when $\lambda_{\mathrm{T}}=0.28$, $\beta_{0}=0.8$. (a) $\mathrm{L} *=0.264$. (b) $\mathrm{L} *=0.403$. (c) $\mathrm{L} *=0.542$.

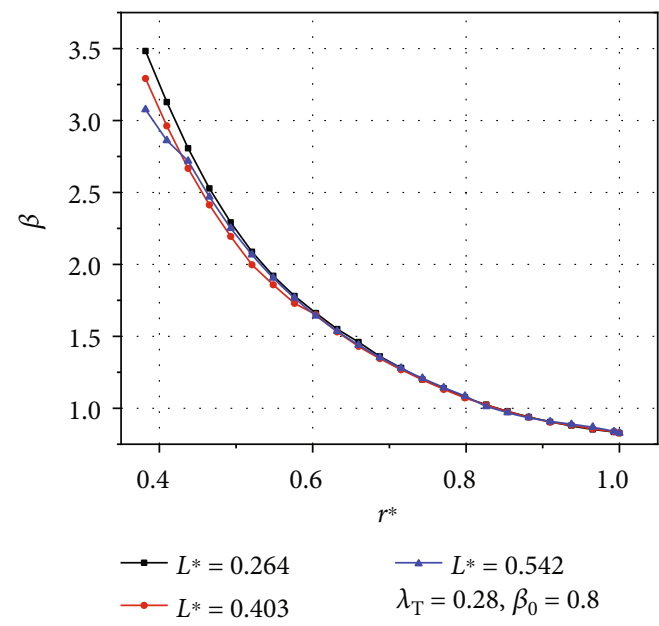

(a) $\beta$

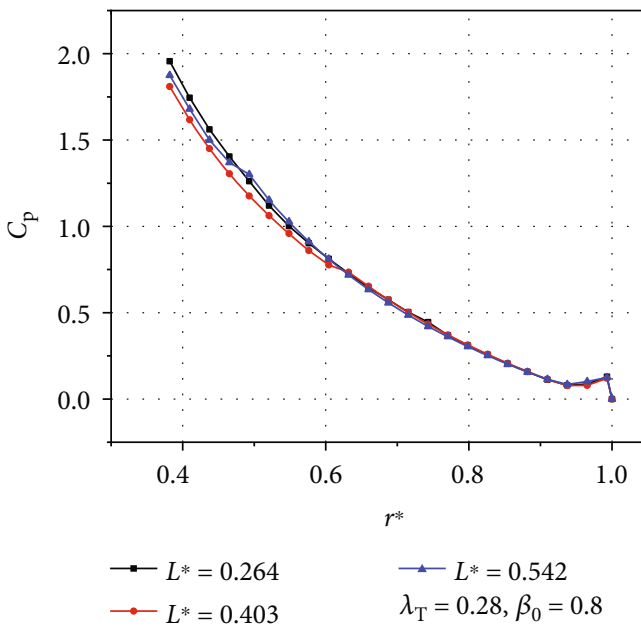

(b) $C_{\mathrm{p}}$

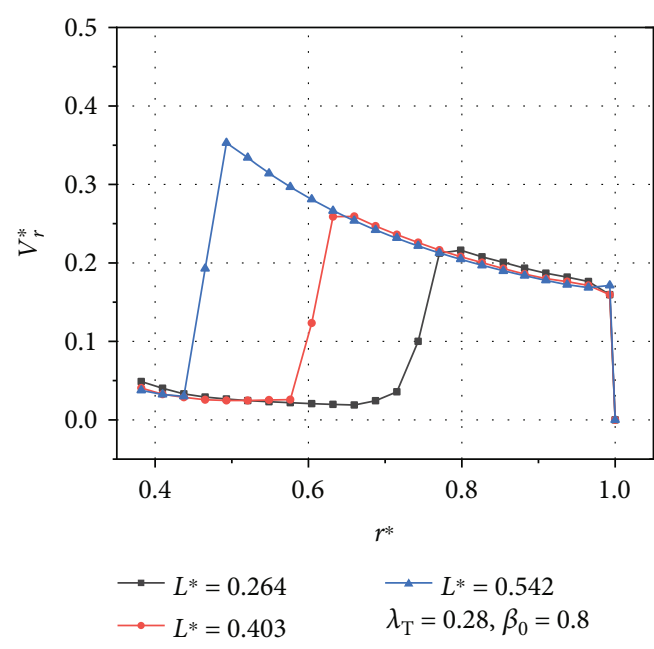

(c) $V_{\mathrm{r}}{ }^{*}$

FIgURE 9: Comparison between the radial distribution of $\beta, C_{\mathrm{p}}$, and $V_{\mathrm{r}}{ }^{*}$ in cavity with different baffles of different $L^{*}$ under the condition $\lambda_{\mathrm{T}}=0.28$ and $\beta_{0}=0.8$. 


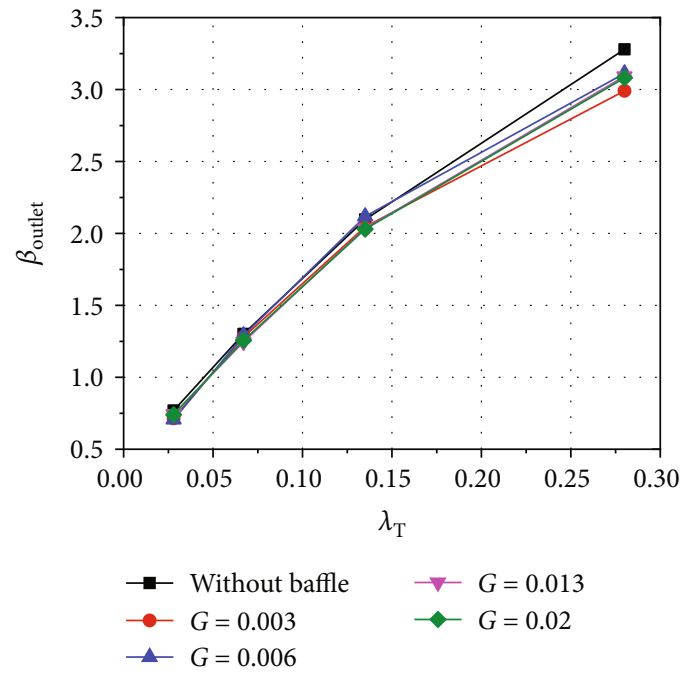

(a) $\beta_{\text {outlet }}$

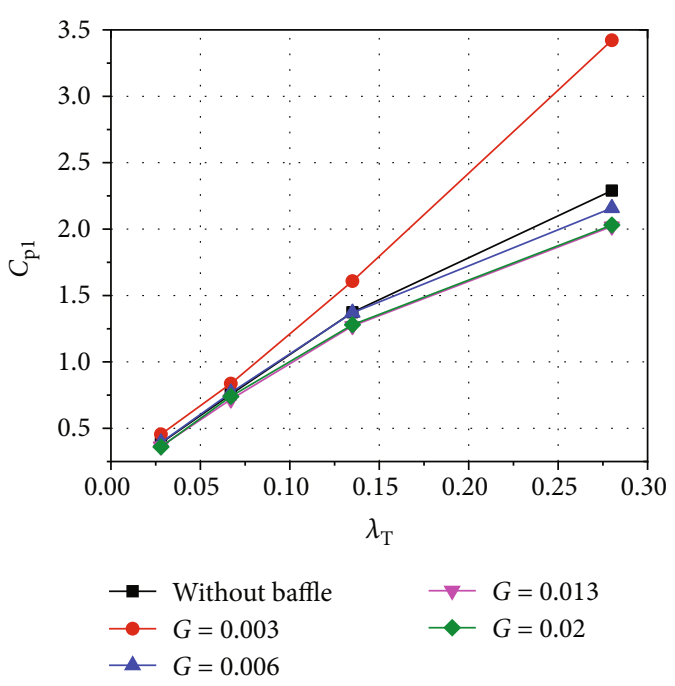

(b) $C_{\mathrm{p} 1}$

FIgURE 10: The variation of $\beta_{\text {outlet }}$ and $C_{\mathrm{p} 1}$ with $\lambda_{\mathrm{T}}$ when $\beta_{0}=0.8$ of the cavity without and with four different baffles of different spaces.

Figures 11(a)-11(d) show the comparison of the radial distribution of the average $\beta, C_{\mathrm{p}}$, nondimensional radial velocity $V_{\mathrm{r}}{ }^{*}$, and total pressure coefficient $C_{\mathrm{pt}}$ in cavity with no baffle and with different baffles of different $G 1$ under the condition $\lambda_{\mathrm{T}}=0.28$ and $\beta_{0}=0.8$. As shown in Figure 11(a), when $G 1=0.02,0.013$, and 0.006 , the distribution of $\beta$ in the cavity is basically the same, when $G 1=0.003$, the variation rate of $\beta$ in the cavity is slowed down because the tangential motion of the airflow is limited by the stationary wall. As shown in Figure 11(b), when $G 1=0.02$ and $G 1=$ 0.013 , the $C_{\mathrm{p}}$ distribution in the cavity is the same and smaller than that in the cavity without baffle; when $G 1$ reduces to 0.006 , although the $\beta$ distribution is basically the same as $G=0.02$ and 0.013 , the variation rate of $C_{\mathrm{p}}$ in the clearance between the baffle and the rotating wall is bigger than $G 1=0.02,0.013$; when $G 1$ continues to decrease to 0.003 , although $\beta$ decreases, $C_{\mathrm{p}}$ increases significantly and is several times of that when $G=0.02,0.013$, and 0.006 .

The reasons for the inconsistency between $\beta$ and $C_{p}$ are as follows. (1) The inward flow in the clearance is an acceleration process. The smaller $G 1$ is, the larger the radial velocity is, and the more obvious the acceleration is, as shown in Figure 11(c). However, the increase of radial velocity will result in a decrease of static pressure. (2) The large radial velocity will cause an increase of the hydraulic friction loss in the radial flow process. As shown in Figure 11(d), as G1 decreases, the total pressure loss in the clearance increases and leads to a decrease in static pressure.

In summary, the variation of the space between the baffle and the rotating wall under small amplitude has little impact on $\beta_{\text {outlet }}$ and $C_{\mathrm{p} 1}$ of the impeller rear cavity; when $G 1$ is reduced to 0.006 or even 0.003 , the large radial velocity will lead to the high total pressure loss caused by the hydraulic friction and the acceleration process of radial inflow leads to a decrease in static pressure because of the increase of the dynamic pressure. These two factors work together, resulting in a large increase in the static pressure loss in the cavity.

\subsection{The Effect of the Swirl-Controlled Orifice}

3.2.1. The Effect on the Flow Structure. The flow structure of the cavity with swirl-controlled orifice under the condition $\lambda_{\mathrm{T}}=0.28, \beta_{0}=0$, and $\mathrm{Ra}=0.2$ is analyzed. As shown in Figure 12, four sections are selected from the cavity to draw the streamlines, swirl ratio contours, and static pressure contours: the $r-z$ section (section A), the section with $z^{*}=0.01$ (the middle yellow section between the rotor and the stator at the highest radius, section $\mathrm{B}$ ), the section with $r^{*}=0.92$ (the green section whose radius is the same as the center of the orifice, section $\mathrm{C}$ ), and the section with $r^{*}=0.55$ (the red section at the middle of the large clearance cavity, section D).

It can be seen from Figure 12 that the jet is formed at the outlet of the orifice and jetted toward the rotating disk at a high speed which will suppress the centrifugal pumping effect and thus thinning the boundary layer of the rotating wall. In the current paper, the orifices on the stator extend axially and tangentially in a direction substantially opposite to the rotating direction of the rotor; thus, the high-speed jet at the exit of the orifice has a high reverse tangential speed. Therefore, when the main flow is mixed with the jet flow, the angular momentum of both pressurized air flows will act on each other and the angular momentum of the total pressurized air contained within the impeller rear cavity will reduce. In the region near the orifice, due to the existence of the complex three-dimensional vortex, the flow is nonaxisymmetric and completely different from the Batchelor flow pattern. As the flow develops inward, the mixing between main flow and jet flow becomes more and more complete; the threedimensional vortex gradually disappears. Meanwhile, the flow structure in the cavity becomes the Batchelor flow 


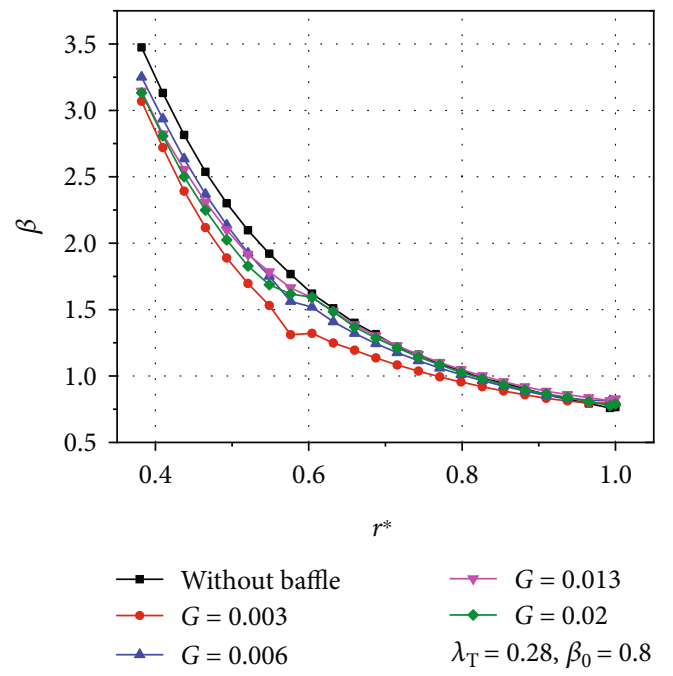

(a) $\beta$

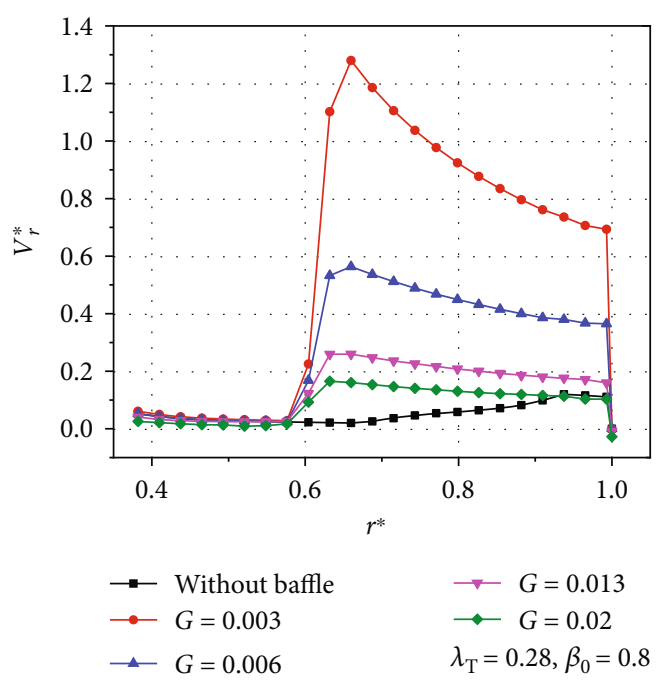

(c) $V_{\mathrm{r}}{ }^{*}$

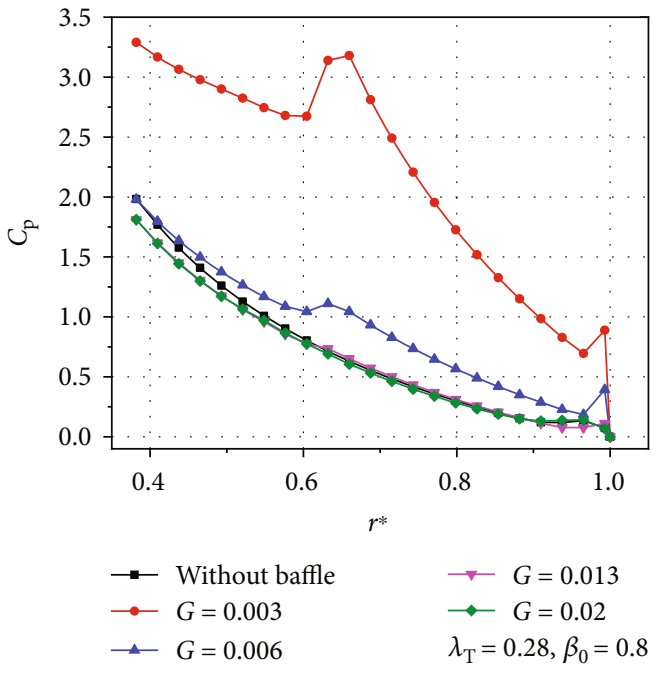

(b) $C_{\mathrm{p}}$

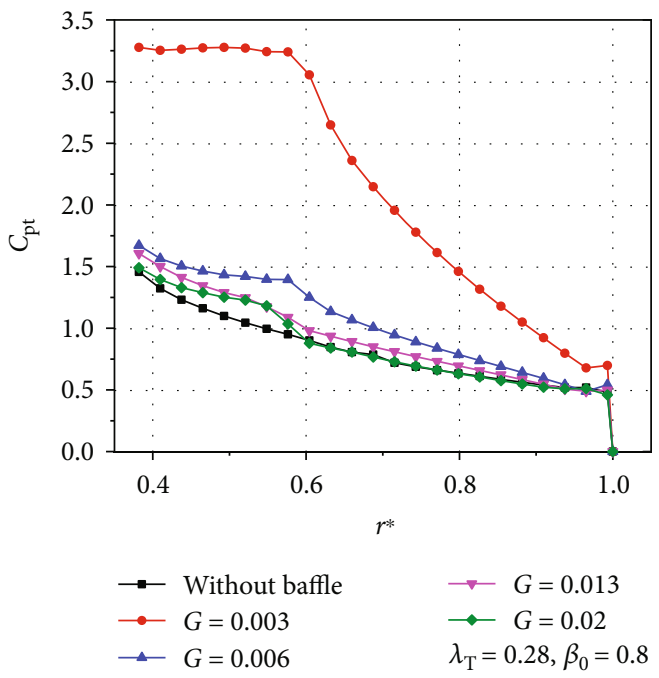

(d) $C_{\mathrm{pt}}$

FIGURE 11: Comparison between the radial variation of $\beta, C_{\mathrm{p}}, V_{\mathrm{r}}{ }^{*}$, and $C_{\mathrm{pt}}$ in cavity without and with different baffles of different $G 1$ under the condition $\lambda_{\mathrm{T}}=0.28$ and $\beta_{0}=0.8$.

pattern again, shown as the swirl ratio and static pressure contours in the $r^{*}=0.55$ section which indicate the swirl ratio and static pressure are substantially constant along the axial direction.

When the mass flow rate ratio $\mathrm{Ra}$ is changed, the flow structure in the impeller rear cavity changes. Figures 13(a) and 13(b) show the swirl ratio contours and streamlines in two sections $\left(z^{*}=0.01\right.$ section and $r^{*}=0.92$ section) of another two conditions. The values of $\lambda_{\mathrm{T}}$ and $\beta_{0}$ of the two conditions are both set as 0.28 and 0.8 , the $\mathrm{Ra}$ are 0.1 and 0.3 , respectively.

Through comparison between Figure 13(a) and Figure 12, both the axial velocity and the tangential velocity of the jet flow at the outlet of the orifice are found to be reduced when $\mathrm{Ra}$ is reduced from 0.2 to 0.1 (the accurate velocities under different $\mathrm{Ra}$ are shown in Table 3). With the decrease of the axial velocity of the jet flow, the influence region of the jet becomes smaller. With the decrease of the tangential velocity, the jet flow with reverse tangential velocity changes the flow direction quickly under the influence of the main flow. As a result, the vortex originally existing in the $z^{*}=0.01$ section disappears, which means the jet depth of the jet flow becomes shallower. The decrease of the tangential velocity and the shallower jet depth caused by the decrease of the jet flow rate increase the swirl ratio in the cavity.

The comparison between Figure 13(b) and Figure 12 shows that both the axial velocity and the tangential velocity of the jet flow increase when Ra increases. Therefore, the jet depth of the jet flow increases, the vortex of the $z^{*}=0.01 \mathrm{sec}-$ tion becomes larger and more complicated, and the radial area affected by the jet correspondingly becomes larger. Because of the above reasons, the swirl ratio in the cavity is greatly reduced, even to a negative value. 


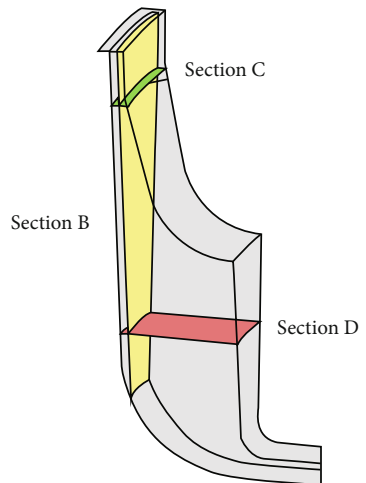

(a)

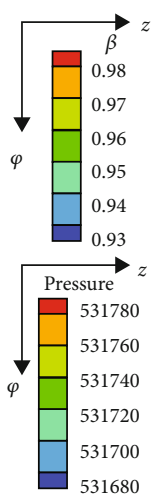

680

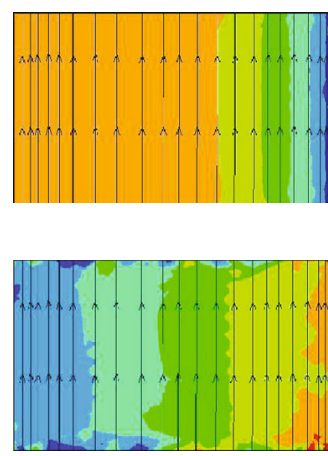

(b)

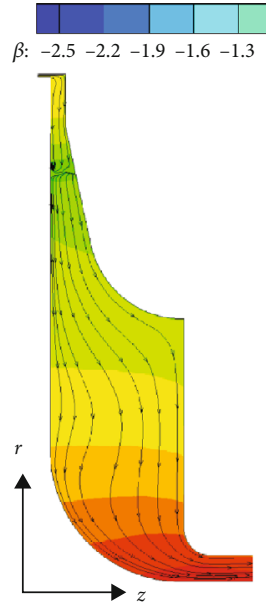

(c)

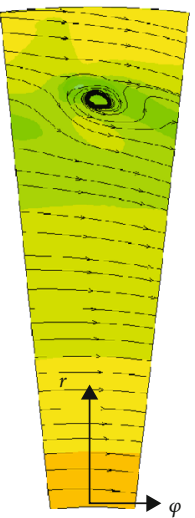

(d)

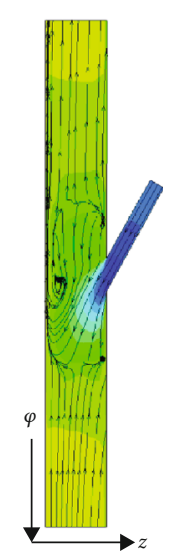

(e)

Figure 12: The swirl ratio and static pressure contours and streamlines in different sections of cavity with swirl-controlled orifice under the condition $\lambda_{\mathrm{T}}=0.28, \beta_{0}=0.8$, and $\mathrm{Ra}=0.2$. (a) Sections schematic. (b) $\mathrm{r} *=0.55$ (section $\mathrm{D}$ ). (c) $\mathrm{r}-\mathrm{z}$ section. (d) $\mathrm{z} *=0.01$ (section $\mathrm{B}$ ). (e) $\mathrm{r} *=0.092$ (section $\mathrm{C}$ ).

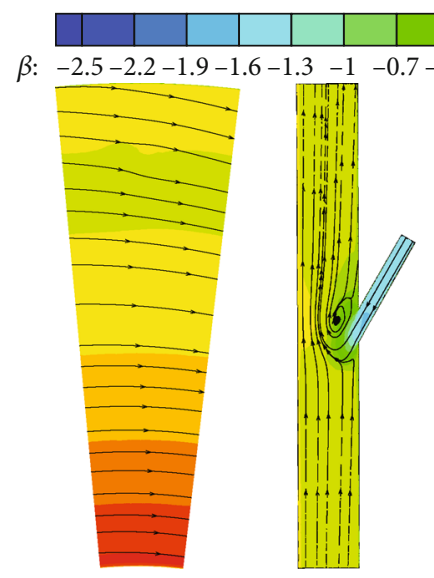

(a)

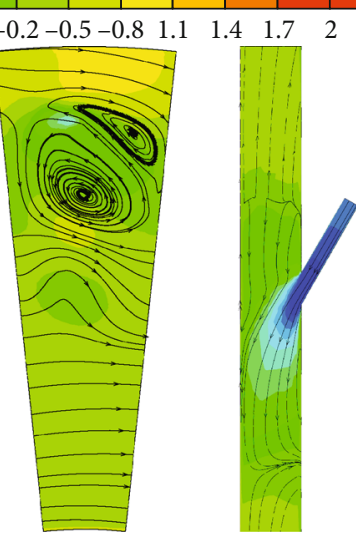

(b)

Figure 13: The swirl ratio contours and streamlines in two sections $\left(z^{*}=0.01\right.$ and $\left.r^{*}=0.92\right)$ under the condition $\lambda_{\mathrm{T}}=0.28, \beta_{0}=0.8$. (a) $\mathrm{Ra}=0.1$. (b) $\mathrm{Ra}=0.3$.

TABLE 3: The velocity at outlet of the orifice under different Ra.

\begin{tabular}{lccc}
\hline Velocity at the outlet of the orifice $(\mathrm{m} / \mathrm{s})$ & 0.1 & $\mathrm{Ra}$ & \\
& 0.2 & 0.3 \\
\hline Total velocity & 218 & 364 & 462 \\
Axial velocity & 110 & 187 & 247 \\
Tangential velocity & 188 & 312 & 390 \\
\hline
\end{tabular}

3.2.2. The Effect on the Pressure Loss. Figures 14(a)-14(d) show the static pressure contours in the rotating disk of the cavity without and with swirl-controlled orifice. As shown in Figure 14(a), the static pressure distribution of the rotating disk is axisymmetric at all the radii when there is no swirlcontrolled orifice in the cavity. However, for the cavity with swirl-controlled orifice shown in Figure 14(b), the static pressure of the rotating disk is nonuniformly distributed in the circumferential direction near the radial position of the ori- fice because of the influence of the jet flow from the orifice. The stagnation of the high-speed jet flow on the rotating disk causes the local high pressure zone, and the complex threedimensional vortex caused by the jet gives rise to the low pressure recirculation zone. With the increase of $\mathrm{Ra}$ from 0.1 to 0.3 , shown in Figures 14(b)-14(d), the influence area of the jet flow from the orifice increases, and thus, the uneven pressure region increases.

However, as the flow develops radially inward, the main flow and the jet flow are fully blended; the static pressure distribution of the rotating wall becomes axisymmetric in the low radius region. It can be seen from Figure 15 that under different conditions, there is a core region with a constant swirl ratio in the $r^{*}=0.55$ section. In addition, the radial velocity of the core zone is relatively small comparing with the tangential velocity. Therefore, it can be considered that the flow satisfies the simple radial equilibrium equation, 


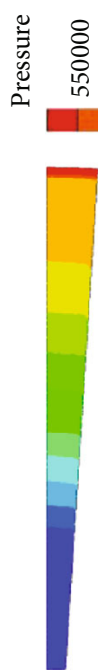

(a)

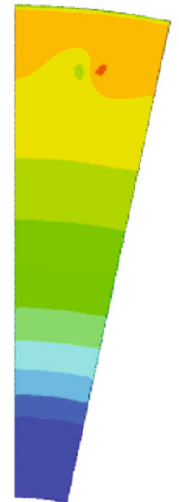

(b)

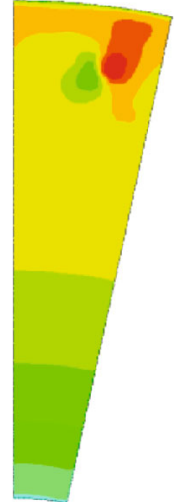

(c)

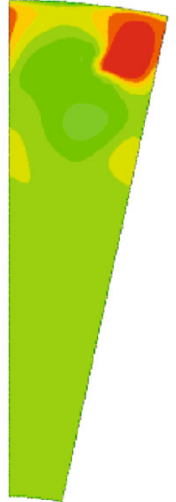

(d)

FIgure 14: The static pressure contours in the rotating disk of the cavity: (a) without orifice; (b) with orifice, $\mathrm{Ra}=0.1$; (c) with orifice, $\mathrm{Ra}=0.2$; (d) with orifice, $\mathrm{Ra}=0.3$.

which means the radial distribution of pressure in the cavity is decided by the central core swirl ratio $\beta$. The above results prove the conclusion made in the flow structure analysis that the flow structure at low radius in the impeller rear cavity with swirl-controlled orifice is of Batchelor type.

Figures 16(a) and 16(b) show the comparisons between the variation of the outlet swirl ratio $\beta_{\text {outlet }}$ and the outlet pressure loss $C_{\mathrm{p} 1}$ with $\beta_{0}$ of the impeller rear cavity with swirl-controlled orifice and the variation of $\beta_{\text {outlet }}$ and $C_{\mathrm{p} 1}$ with $\beta_{0}$ of the cavity without orifice.

It can be seen from Figure 16(a) that $\beta_{\text {outlet }}$ increases with the increase of $\beta_{0}$ regardless of whether there is swirlcontrolled orifice in the cavity. When $\beta_{0}$ is in the range of $0 \sim 0.8, \beta_{\text {outlet }}$ of the cavity with swirl-controlled orifice is smaller than that of the cavity without orifice and decreases as $\mathrm{Ra}$ increases. For the cases whose $\mathrm{Ra}$ increases to 0.3 , $\beta_{\text {outlet }}$ decreases to a negative value for low $\beta_{0}$ conditions.

It can be seen from Figure 16(b) that $C_{\mathrm{p} 1}$ of the cavity with swirl-controlled orifice is smaller than that of the cavity without orifice and decreases with increasing $\mathrm{Ra}$ when $\mathrm{Ra}$ is in the range of $0.1 \sim 0.2, \beta_{0}$ is in the range of $0 \sim 0.8$. However, when Ra increases to 0.3 , although $C_{\mathrm{p} 1}$ on the condition with high $\beta_{0}$ is still smaller than that of the condition with $\mathrm{Ra}=$ $0.2, C_{\mathrm{p} 1}$ on the condition with low $\beta_{0}$ is bigger than that of the condition with $\mathrm{Ra}=0.2$.

The reason for the inconsistency between the variations of $C_{\mathrm{p} 1}$ and $\beta_{\text {outlet }}$ with $\beta_{0}$, for the conditions with $\mathrm{Ra}=0.3$, $\beta_{0}<0.4$, is that the flow at the low radius, where it is away from the orifice, satisfies the simple radial equilibrium equation $-d p^{*} / d r^{*}=2 \beta^{2} r^{*}$, which means the radial distribution of pressure in the cavity is decided by the absolute value distribution of central core swirl ratio $\beta$. When $\mathrm{Ra}=0.3$, $\beta_{0}<0.4$, although $\beta_{\text {outlet }}$ is reduced (reduced to a negative value), its absolute value is actually increased (shown in Figure 16(a)), and thus, $C_{\mathrm{p} 1}$ increases.

Because of the important impact of the static pressure distribution in the cavity on the high-pressure rotating assembly axial load, further researches of the static pressure and swirl ratio distributions have been done.

Figures 17 and 18 show the contrasts of the radial distribution of swirl ratio and average static pressure in the cavity with and without swirl-controlled orifice on the same conditions. Figures 17 and 18 show the comparisons of cases with $\lambda_{\mathrm{T}}=0.28$ and $\beta_{0}=0.8,0$, respectively. As shown in Figure 17, when $\beta_{0}=0.8$, due to the mixing effect of the reverse swirling jet flow at the outlet of the orifice, the main flow $\beta$ is smaller than that of the cavity without orifice, and the larger $\mathrm{Ra}$ is, the lower main flow $\beta$ is. Thus, the decrease of the main flow $\beta$ leads to the reduction of $C_{\mathrm{p}}$. In addition, the flow is very complicated due to the strong influence of the jet at the exit of the orifice when $\mathrm{Ra}$ is large and thus causes the fluctuation of the radial distribution of $\beta$ and the drastic increase of local $C_{\mathrm{p}}$ . However, as the flow develops radially inward, the vorticity gradually disappears and $C_{\mathrm{p}}$ decreases.

As shown in Figure 18 , when $\beta_{0}=0$, the main flow $\beta$ is also reduced under the influence of the jet, and the larger $\mathrm{Ra}$ is, the lower main flow $\beta$ is. The difference between the condition with $\beta_{0}=0$ and the condition with $\beta_{0}=0.8$ is that under the influence of the jet, main flow $\beta$ becomes a negative value and will decrease as the flow develops radially inwards (which means the increase of the absolute value of $\beta$ ) when $\mathrm{Ra}$ is large. Therefore, the increase in the absolute value of the main flow $\beta$ leads to the increase of $C_{\mathrm{p}}$.

3.2.3. The Influence of Geometry Parameters of the SwirlControlled Orifice. The swirl-controlled orifice arranged on the stationary wall introduces the reverse swirling flow to mix with the high swirling main flow so that the swirl ratio of the main flow is greatly reduced and the deswirl effect is achieved. For swirl-controlled orifice, the geometry parameters which will influence the deswirl effect include the number, length, diameter, orientation, and radial position. In the current paper, the angle between the orifice axial and the tangential direction and the radial position are discussed to investigate their effects on the swirl ratio and static pressure distributions in the cavity.

(1) The Orientation. Figures 19(a) and 19(b) show the variation of $\beta_{\text {outlet }}$ and $C_{\mathrm{p} 1}$ with $\beta_{0}$ when $\lambda_{\mathrm{T}}=0.28, \mathrm{Ra}=0.2$ of the cavity without and with three different swirl-controlled orifices whose angle $\alpha$ between the axial and the tangential directions is $30^{\circ}, 45^{\circ}$, and $60^{\circ}$, respectively. For all the cavities, the change rule of $C_{\mathrm{p} 1}$ with $\beta_{0}$ is the same as that of $\beta_{\text {outlet }}$ with $\beta_{0}$; when $\beta_{0}$ is small, $\alpha$ increases from $30^{\circ}$ to $45^{\circ}, \beta_{\text {outlet }}$ increases, $\alpha$ continues to increase from $45^{\circ}$ to $60^{\circ}$, and $\beta_{\text {outlet }}$ is basically unchanged; when $\beta_{0}$ is large, $\beta_{\text {outlet }}$ gradually increases with increasing $\alpha$.

Figures 20(a) and 20(b) show contrasts of the radial distribution of the average $\beta$ and $C_{\mathrm{p}}$ in the cavity without and with different orifices of different orientations under 

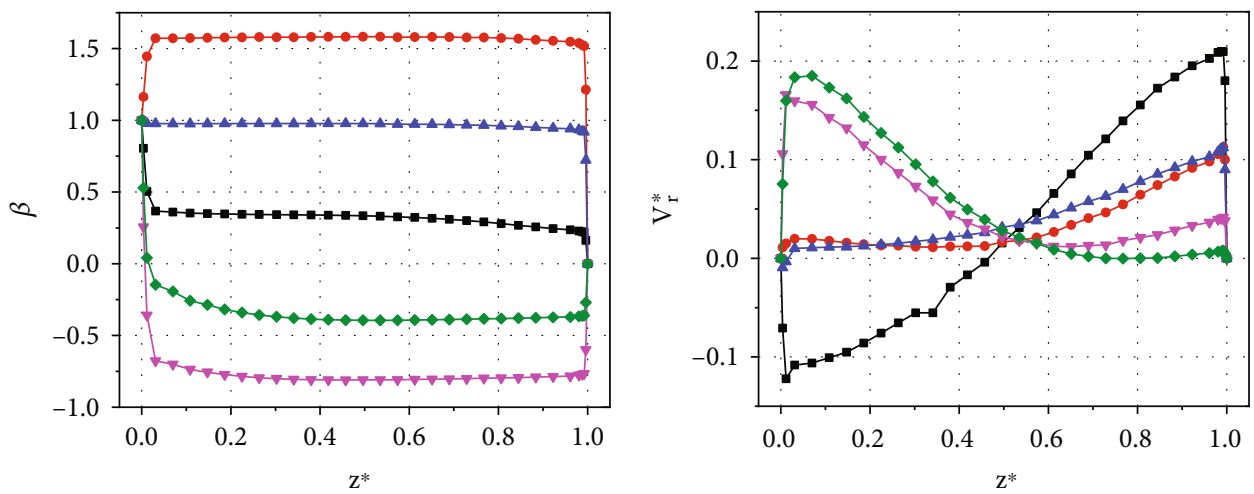

$$
\begin{aligned}
& \rightarrow \mathrm{Ra}=0.1, \beta_{0}=0.8 \quad \rightarrow \mathrm{Ra}=0.3, \beta_{0}=0 \\
& \rightarrow \mathrm{Ra}=0.2, \beta_{0}=0.8 \quad \rightarrow \mathrm{Ra}=0.3, \beta_{0}=0.4 \\
& \rightarrow \mathrm{Ra}=0.1, \beta_{0}=0
\end{aligned}
$$

FIgURE 15: The axial variations of swirl ratio and radial velocity at $r^{*}=0.55$ radius under conditions with different Ra and $\beta_{0}$.

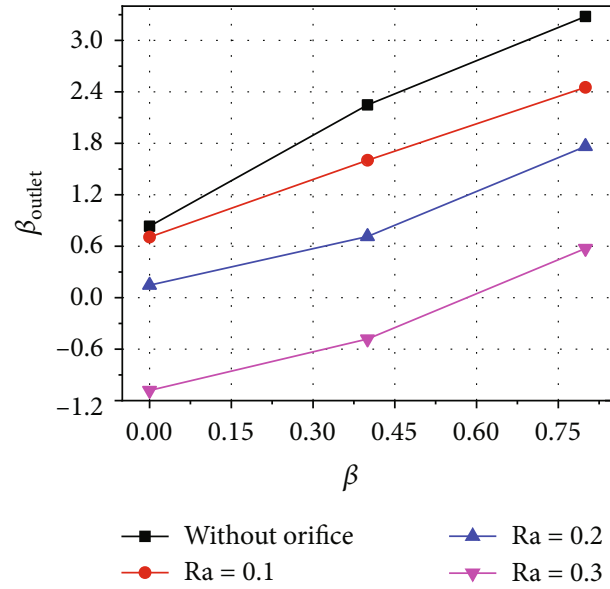

(a) $\beta_{\text {outlet }}$

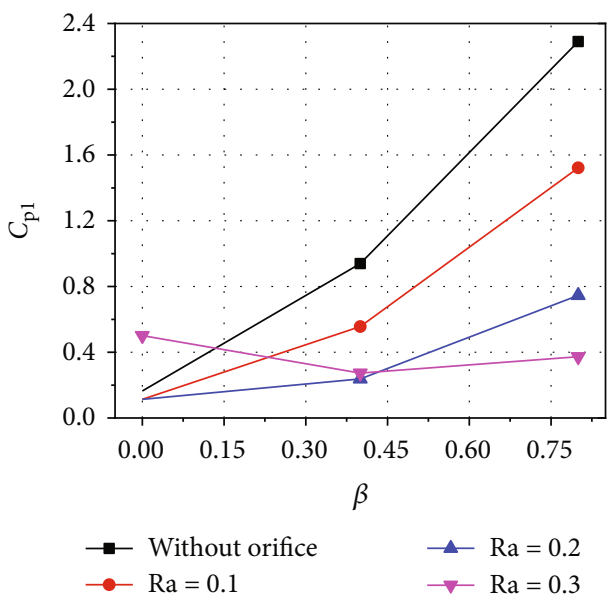

(b) $C_{\mathrm{p} 1}$

FIGURE 16: The comparisons between the variations of $\beta_{\text {outlet }}$ and $C_{\mathrm{p} 1}$ with $\beta_{0}$ of the impeller rear cavity with swirl-controlled orifice and those of the cavity without orifice.

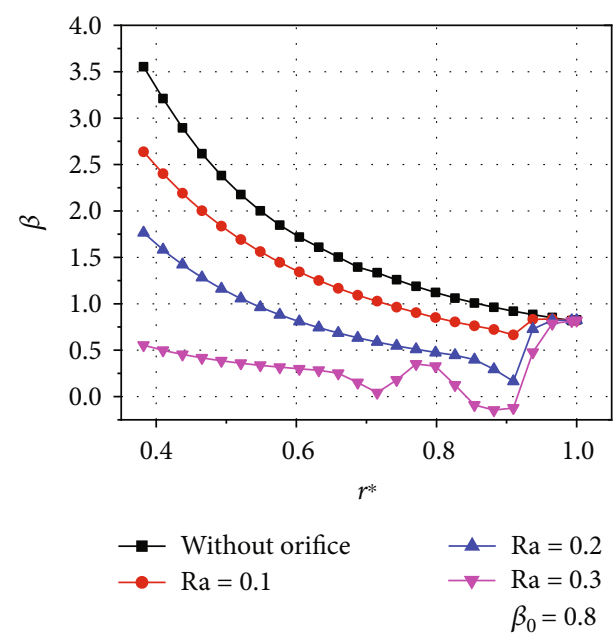

(a) $\beta$

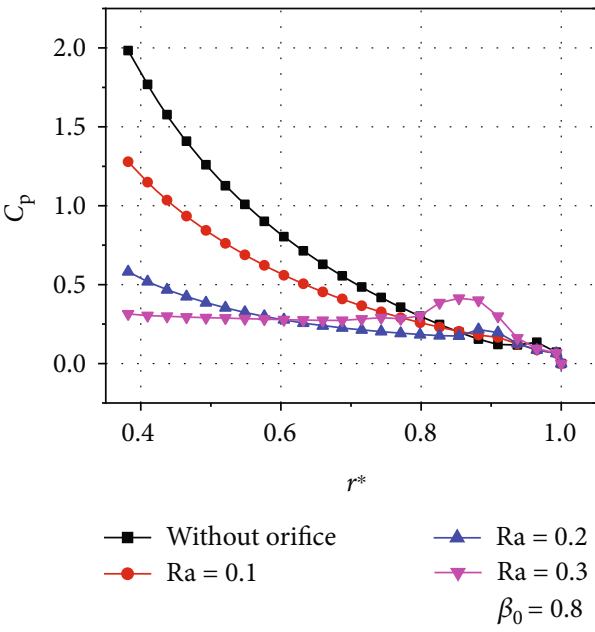

(b) $C_{\mathrm{p}}$

FIGURE 17: The contrasts of the radial distribution of average static pressure and $\beta$ in the cavity with and without swirl-controlled orifice when $\lambda_{\mathrm{T}}=0.28$ and $\beta_{0}=0.8$. 


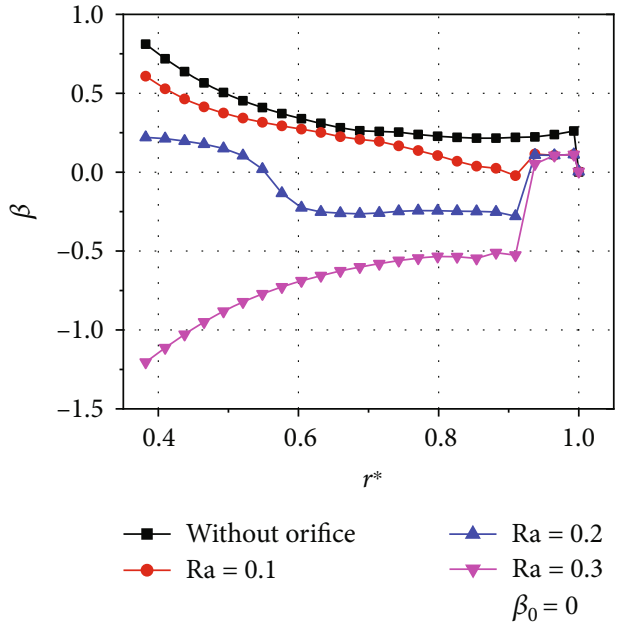

(a) $\beta$

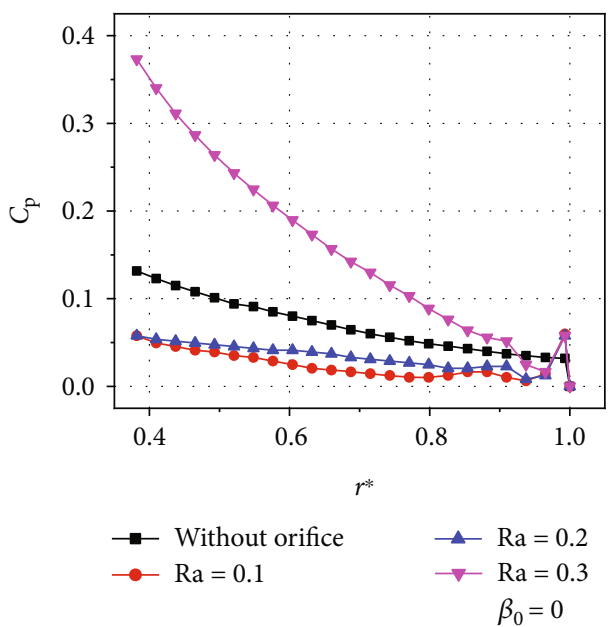

(b) $C_{\mathrm{p}}$

FiguRE 18: The contrasts of the radial variation of average static pressure and $\beta$ in the cavity with and without swirl-controlled orifice when $\lambda_{\mathrm{T}}=0.28$ and $\beta_{0}=0$.

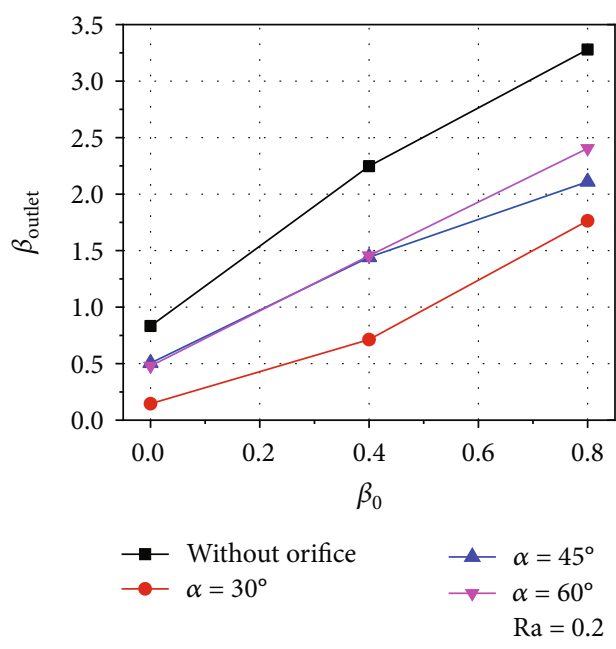

(a) $\beta_{\text {outlet }}$

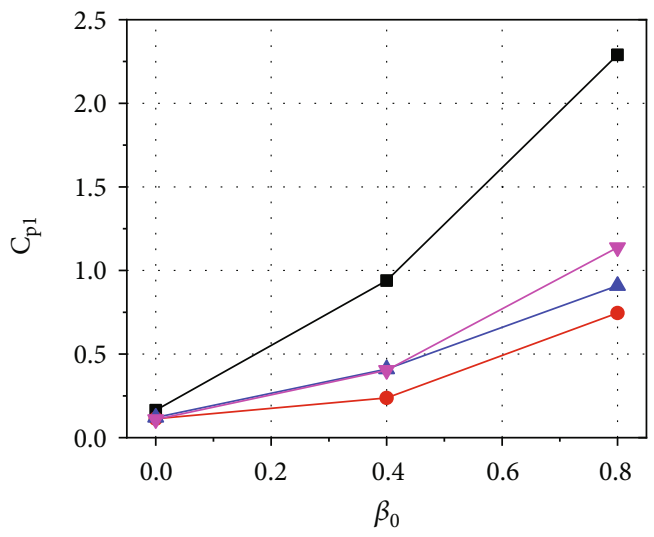

$$
\begin{array}{ll}
\longrightarrow & \text { Without orifice } \\
\rightarrow \alpha=30^{\circ} & \longrightarrow \alpha=45^{\circ} \\
& \checkmark \alpha=60^{\circ}
\end{array}
$$

(b) $C_{\mathrm{p} 1}$

Figure 19: The variation of $\beta_{\text {outlet }}$ and $C_{\mathrm{p} 1}$ with $\beta_{0}$ of the impeller rear cavity without and with three different orifices whose $\alpha$ is $30^{\circ}, 45^{\circ}$, and $60^{\circ}$, respectively.

the condition $\mathrm{Ra}=0.28$ and $\beta_{0}=0.4$. When there is a swirlcontrolled orifice, $\beta$ near the orifice shows a sudden drop. When $\alpha$ is $30^{\circ}, \beta$ decreases the most; when $\alpha=45^{\circ}$ and $\alpha=$ $60^{\circ}, \beta$ decreases substantially the same, which eventually leads to the variation law of $\beta_{\text {outlet }}$ as shown in Figure 19(a). However, for the radial variation of $C_{p}$ shown in Figure 20(b), $C_{p}$ near the orifice increases instead because of the pressure loss of the mixing process. When $\alpha=30^{\circ}$, the area affected by the jet is significantly larger than that of $\alpha=45^{\circ}$ and $\alpha=60^{\circ}$, and $C_{\mathrm{p}}$ is larger; as the flow progresses downward, the mixing is completed, and the flow field eventually becomes uniform. At this time, the radial variation of $C_{\mathrm{p}}$ is determined by $\beta$, so $C_{\mathrm{p} 1}$ and $\beta_{\text {outlet }}$ change in the same manner.
Figures 21(a)-21(c) show the streamlines and velocity contours in two sections $\left(z^{*}=0.01\right.$ section and $r^{*}=0.92$ section) of the cavity with three different orifices of three orientations $30^{\circ}, 45^{\circ}$, and $60^{\circ}$. It can be seen from the figure that when $\mathrm{Ra}$ is the same, the larger the angle is, the smaller the tangential velocity of jet flow is and the larger the axial velocity is. The decrease of the tangential velocity of the jet causes the weakening of the deswirl effect; the increase of the axial velocity of the jet causes more outlet flow of the orifice to be sprayed and stagnated on the rotating wall which means the influence of the jet on the mainstream is weakened. However, for the condition of $\mathrm{Ra}=0.2$ and $\beta_{0}=0.4$, when the angle increases to $45^{\circ}$, the jet flow has already all been sprayed on the rotating wall, the increasing angle (from $45^{\circ}$ to $60^{\circ}$ ) has little effect on the flow field. 


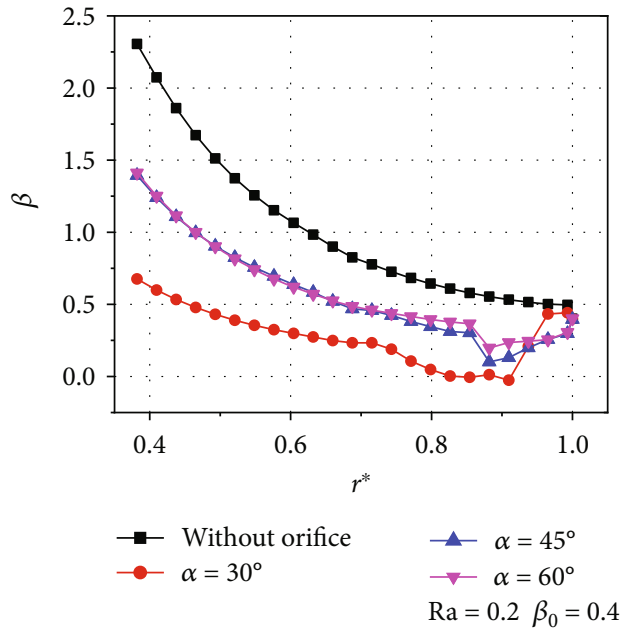

(a) $\beta$

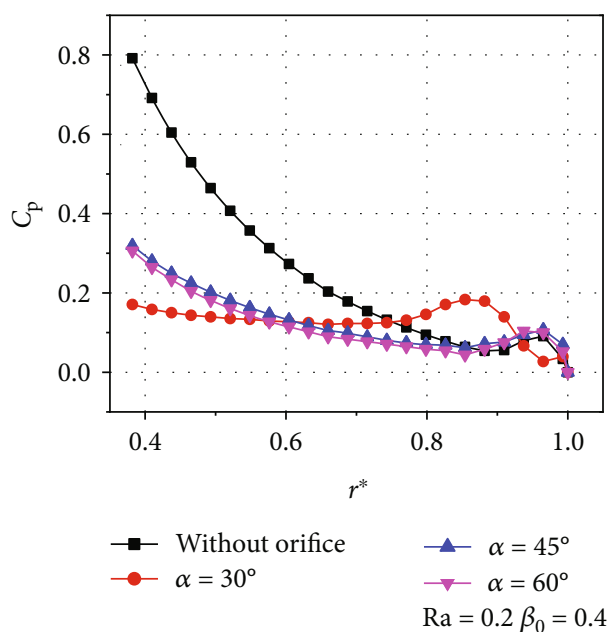

(b) $C_{\mathrm{p}}$

Figure 20: The contrasts between the radial distribution of $\beta$ and $C_{\mathrm{p}}$ in cavity without and with different orifices of different $\alpha$ under the condition $\mathrm{Ra}=0.2$ and $\beta_{0}=0.8$.
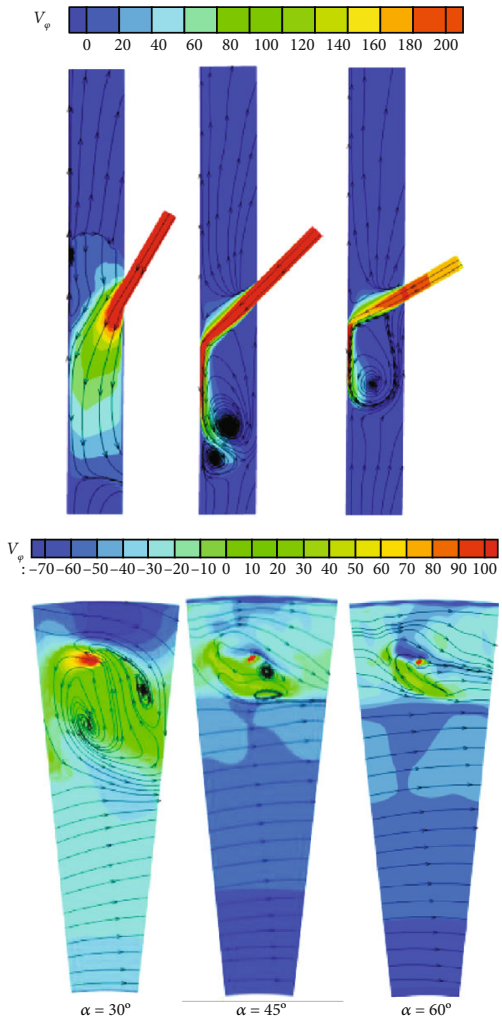

(a)
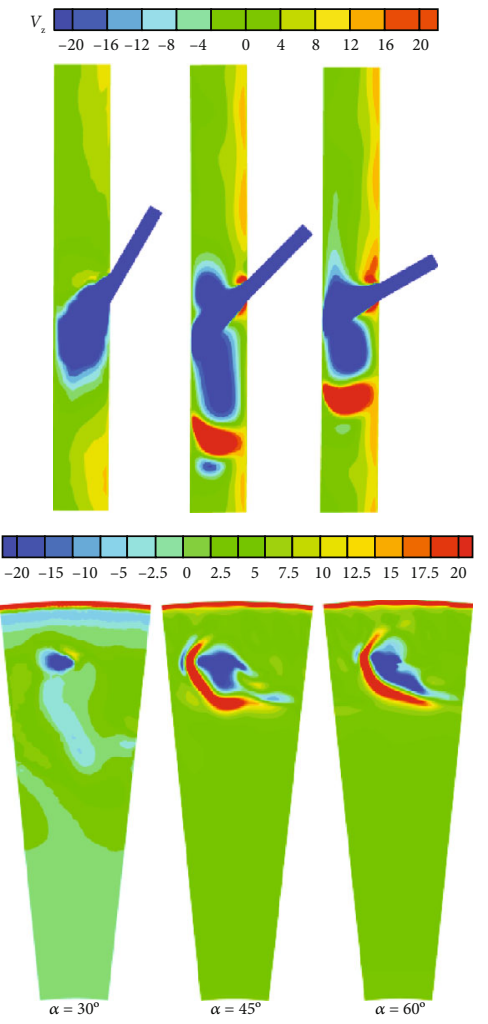

(b)
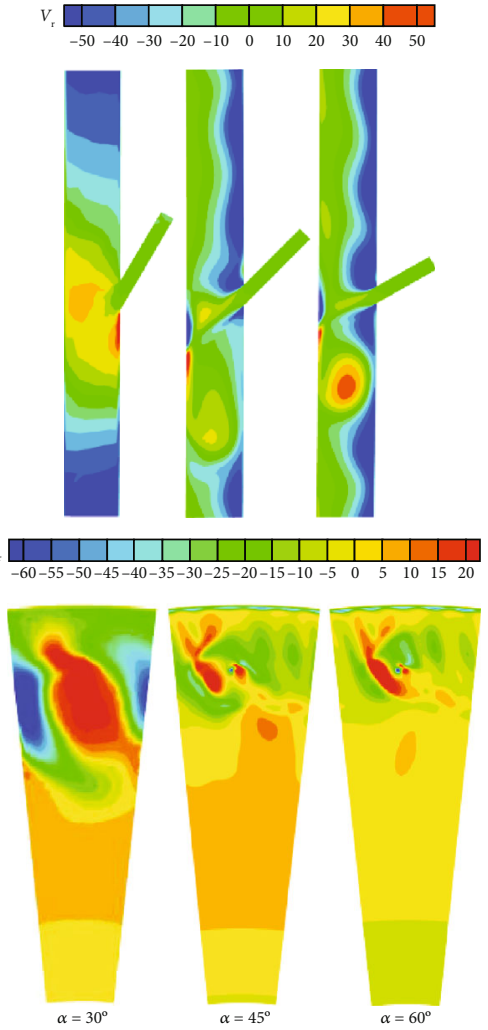

(c)

FIGURE 21: The velocity contours and streamlines in two sections $\left(z^{*}=0.01\right.$ and $\left.r^{*}=0.92\right)$ under the condition $\mathrm{Ra}=0.2, \beta_{0}=0.4$.

In summary, when Ra is equal and the angle of the swirlcontrolled orifice is small, the tangential velocity of the jet flow decreases as the angle increases, the deswirl effect of the jet on the mainstream is weakened; therefore, $\beta_{\text {outlet }}$ and $C_{\mathrm{p} 1}$ gradually increase with the increase of angle. When the angle increases to a certain value, the jet flow has already all sprayed on the rotating wall; $\beta_{\text {outlet }}$ and $C_{\mathrm{p} 1}$ are basically unchanged with increasing angle.

(2) Radial Position. Figures 22(a) and 22(b) show the variation of $\beta_{\text {outlet }}$ and $C_{\mathrm{p} 1}$ with $\beta_{0}$ when $\lambda_{\mathrm{T}}=0.28, \mathrm{Ra}=0.1$, without and with four different swirl-controlled orifices whose 


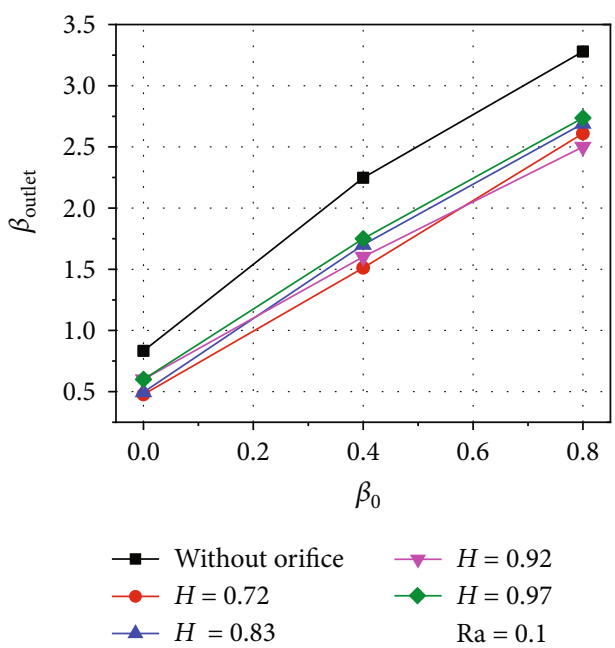

(a) $\beta_{\text {outlet }}$

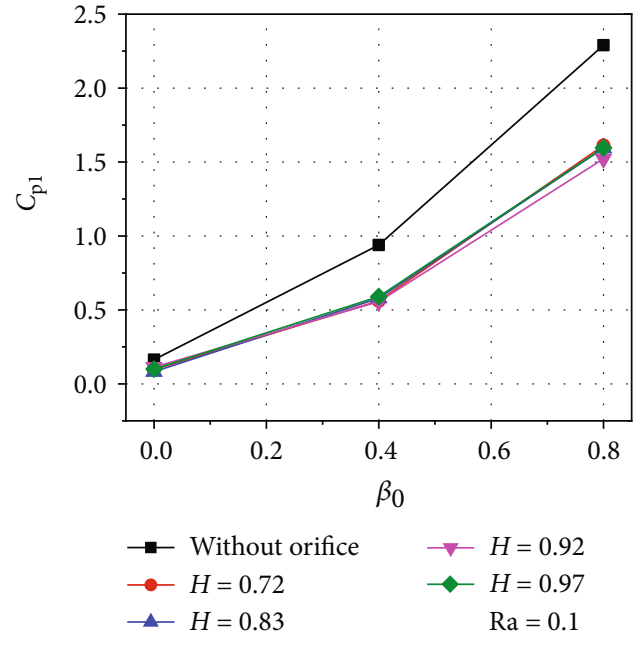

(b) $C_{\mathrm{p} 1}$

FIGURE 22: The variation of $\beta_{\text {outlet }}$ and $C_{\mathrm{p} 1}$ with $\beta_{0}$ of the impeller rear cavity without and with four different orifices whose radial position $H$ is $0.72,0.83,0.92$, and 0.97 , respectively.

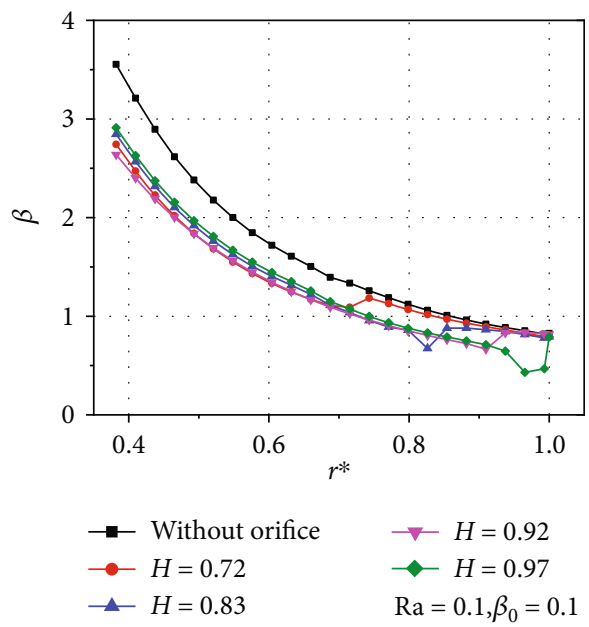

(a) $\beta$

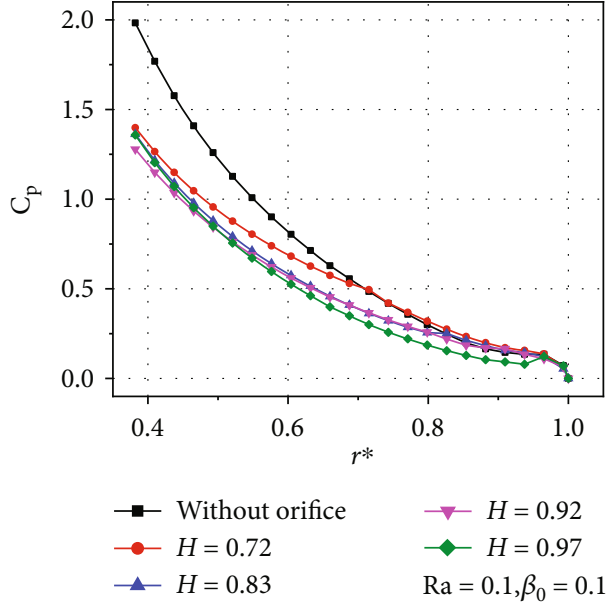

(b) $C_{\mathrm{p}}$

FIgURE 23: Comparison between the radial distribution of $\beta$ and $C_{\mathrm{p}}$ in cavity without and with different orifices of different $H$ under the condition $\mathrm{Ra}=0.1$ and $\beta_{0}=0.8$.

nondimensional radial position $H=R_{\mathrm{O}} / R$ is $0.72,0.83,0.92$, and 0.97 , respectively. It can be seen from the figure that when $\mathrm{Ra}=0.1$ and $\beta_{0}$ is in the range of $0 \sim 0.8$, the radial position $H$ of the swirl-controlled orifice has little impact on $\beta_{\text {outlet }}$ and $C_{\mathrm{p} 1}$. Under different $H, \beta_{\text {outlet }}$ and $C_{\mathrm{p} 1}$ are basically equal.

Figures 23(a) and 23(b) show contrasts of the radial distribution of the average $\beta$ and $C_{\mathrm{p}}$ in cavity with no orifice and with four different orifices of different $H$ under the condition of $\mathrm{Ra}=0.1$ and $\beta_{0}=0.8$. The figure shows that for the cavity with swirl-controlled orifice, the radial distribution of $\beta$ and $C_{p}$ of mainstream in the area before reaching the region near the orifice is the same as that of the cavity without orifice. After reaching the region near the orifice, $\beta$ and $C_{p}$ of the mainstream appear to suddenly drop because of the impact by the jet from the orifice. Whether the radial position of the orifice is high or low, the radial distribution of $\beta$ of the main flow after the mixing of the mainstream and the jet is substantially the same, so $\beta_{\text {outlet }}$ and $C_{\mathrm{p} 1}$ are substantially equal under different $H$. For the cavity with different orifices of different $H$, the unmixed area from the inlet to the orifice increases as $H$ decreases, because the static pressure loss in this area is the same as that of the cavity without orifice; therefore, although $H$ has no effect on $C_{p 1}$, it will change the static pressure distribution at the high radius of the rotating wall, thus changing the axial load of the rotor assembly. As $H$ decreases, the axial load of the rotor assembly decreases. 
In summary, when $\mathrm{Ra}$ is equal, regardless of the radial position of the swirl-controlled orifice, the radial distribution of main flow swirl ratio after mixing is the same, the radial position has little effect on $\beta_{\text {outlet }}$ and $C_{\mathrm{p} 1}$. However, with the decrease of $H$, the unmixed high static pressure loss area between the inlet and the orifice increases, and the axial load of the rotor assembly decreases. Therefore, if the axial load of the rotor assembly needs to be adjusted without changing the outlet static pressure of the cavity, the adjustment of the radial position of the swirl-controlled orifice is a way to achieve it.

\section{Conclusion}

Two control methods, namely, baffle and swirl-controlled orifice, were proposed to regulate the pressure loss and distribution in an impeller rear cavity in the author's previous [15] and the current papers. The numerical simulations were carried out to investigate the control mechanisms of these two methods. The control mechanism of the baffle is that the existence of the big counterclockwise vortex leads to the diminution of the average swirl ratio of cavity [15]. Thus, the pressure loss of cavity with baffle reduces.

The swirl-controlled orifice which could deswirl the flow is more effective in regulating the pressure loss and its distribution in impeller rear cavity than baffle. The control mechanism of the swirl-controlled orifice is that the main air swirl ratio is directly reduced by introducing a jet which is from the combustion section and formed by the inclined orifice with a high reverse tangential velocity. However, more attention should be paid on the detailed design of the orifice, for the reason that too much jet flow will reduce the main flow swirl ratio to a negative value whose absolute value might be bigger than that of the cavity without orifice and thus causes the increase of the pressure loss.

Furthermore, the influence of geometry parameters of the two methods including the length of baffle, the space between the baffle and rotating disk wall, the orientation, and radial position of swirl-controlled orifice was investigated. Through the researches, the main conclusions are made as follows.

When designing the baffle, there is an optimal length to make the static pressure loss in the cavity the smallest. The small amplitude variation of the space between the baffle and the rotating wall and the radial position of the swirlcontrolled orifice have little effect on the outlet static pressure loss of the cavity; however, the radial position of the swirlcontrolled orifice will change the radial distribution of the static pressure on the rotating wall.

\section{Nomenclature}

$R, R_{\mathrm{H}}$ : Inner and outer radius, respectively

S1: $\quad$ The minimum axial spacing between disks

S2: $\quad$ The maximum axial spacing between disks

$R_{\mathrm{B}}$ : Inner radius of baffle

$L^{*}: \quad$ Nondimensional length $=1-R_{\mathrm{B}} / R$

$R_{\mathrm{O}}$ : The radius of axis of the orifice

$D: \quad$ The diameter of the orifice

$X$ : The number of the orifice $\alpha: \quad$ The angle between the axial direction of the orifice and the tangential direction

$H$ : $\quad$ The nondimensional radial position

Ra: The mass flow rate ratio of inlet 2 and inlet 1

$N$ : $\quad$ Rotational speed of impeller

$m$ : Outlet mass flow rate

$r$ : $\quad$ Radius coordinate

$z$ : Axial coordinate

$\Omega: \quad$ Disk angular velocity

$\rho: \quad$ Density

$\mu$ : $\quad$ Dynamic viscosity

$V_{\varphi}: \quad$ Tangential velocity

$V_{\varphi, 0}: \quad$ Inlet tangential velocity

$V_{\mathrm{r}}: \quad$ Radial velocity

$V_{\mathrm{r}}{ }^{*}: \quad$ Nondimensional radial velocity $=V_{\mathrm{r}} / \Omega r$

$\beta: \quad$ Swirl ratio $=V_{\varphi} / \Omega r$

$\beta_{0}: \quad$ Inlet swirl ratio $=V_{\varphi, 0} / \Omega r_{0}$

$P$ : $\quad$ Static pressure

$P_{0}: \quad$ Inlet static pressure

$P_{1}$ : Out static pressure

$C_{\mathrm{w}}: \quad$ Dimensionless mass flow $=m / M r$

$\operatorname{Re}_{\varphi}: \quad$ Rotational Reynolds number $=\rho \Omega R^{2} / \mu$

$\lambda_{\mathrm{T}}: \quad$ Turbulent flow parameter $=C_{\mathrm{w}} / \operatorname{Re}_{\varphi}{ }^{0.8}$

$C_{\mathrm{p}}: \quad$ Pressure coefficient $=2\left(P_{0}-P\right) /\left(\rho \Omega^{2} R^{2}\right)$

$C_{\mathrm{pt}}: \quad$ Total pressure coefficient

$C_{\mathrm{p} 1}$ : Outlet pressure coefficient $=2\left(P_{0}-P_{1}\right) /\left(\rho \Omega^{2} R^{2}\right)$.

\section{Data Availability}

The data used to support the findings of this study are included within the article.

\section{Conflicts of Interest}

The authors declare that they have no conflicts of interest.

\section{Acknowledgments}

The authors gratefully acknowledge the fund from the National Natural Science Foundation of China (approval serial number 51776200) and its approval to publish the current paper.

\section{References}

[1] R. Debuchy, A. Dyment, H. Muhe, and P. Micheau, "Radial inflow between a rotating and a stationary disc," European Journal of Mechanics-B/Fluids, vol. 17, no. 6, pp. 791-810, 1998.

[2] R. Debuchy, F. Abdel Nour, and G. Bois, "On the flow behavior in rotor-stator system with superposed flow," International Journal of Rotating Machinery, vol. 2008, Article ID 719510, 10 pages, 2008.

[3] J. Kurokawa and T. Toyokura, "Study on axial thrust of radial flow turbomachinery," Japan Society of Mechanical Engineers, vol. 2, p. 31, 1972.

[4] X. Liu and K. V. Patel, "A CFD analysis of the flow in impeller rear cavity of aeroengines," in ASME 1993 International Gas 
Turbine and Aeroengine Congress and Exposition, Mississauga, Ontario, Canada, 1993.

[5] S. Poncet, M. P. Chauve, and P. GalLe, "Turbulent rotating disk flow with inward throughflow," Journal of Fluid Mechanics, vol. 522, pp. 253-262, 2005.

[6] S. Poncet, M. P. Chauve, and R. Schiestel, "Batchelor versus Stewartson flow structures in a rotor-stator cavity with throughflow," Physics of Fluids, vol. 17, no. 7, 2005.

[7] Z. EI-Oun, P. H. Meller, and A. B. Turner, "Sealing of a shrouded rotor-stator system with pre-swirl coolant," in ASME 1987 International Gas Turbine Conference and Exhibition, Anaheim, California, USA, May 1987.

[8] J. R. Pincombe, "Flow visualization and velocity measurements in a rotor-stator system with a forced radial inflow," Report 88/TFMRC/TN61, University of Sussex, England, 1988.

[9] K. J. Hart and A. B. Turner, "Influence of radial inflow on rotor-stator cavity pressure distributions," in ASME 1994 International Gas Turbine and Aeroengine Congress and Exposition, The Hague, Netherlands, Jun 1994.

[10] K. J. Hart and A. B. Turner, "Simple design methods for the prediction of radial static pressure distribution in a rotorstator cavity with radial inflow," in ASME 1995 International Gas Turbine and Aeroengine Congress and Exposition, Houston, Texas, USA, Jun 1995.

[11] J. W. Chew, P. R. Farthing, J. M. Owen, and B. Stratford, "The use of fins to reduce the pressure drop in a rotating cavity with a radial inflow," Journal of Turbomachinery, vol. 111, no. 3, pp. 349-356, 1989.

[12] P. R. Farthing, J. W. Chew, and J. M. Owen, "The use of de-swirl nozzles to reduce the pressure drop in a rotating cavity with a radial inflow," in ASME 1989 International Gas Turbine and Aeroengine Congress and Exposition, pp. 106-114, Toronto, Ontario, Canada, Jun 1989.

[13] D. May, J. W. Chew, and T. J. Scanlon, "Prediction of deswirled radial inflow in rotating cavities with hysteresis," Journal of Turbomachinery, vol. 135, no. 4, 2013.

[14] N. Dimitrie and P. Michael, "Secondary air system in aeroengines employing vortex reducers," in ASME Turbo Expo 2001: Power for Land, Sea, and Air, New Orleans, Louisiana, USA, Jun 2001.

[15] G. Liu, Q. Du, J. Liu, P. Wang, and J. Q. Zhu, "Numerical investigation of radial inflow in the impeller rear cavity with and without baffle," Science China Technological Sciences, vol. 59, no. 3, pp. 456-467, 2016. 